\title{
Immunotherapy of COVID-19: Inside and Beyond IL-6 Signalling
}

\author{
Gaetano Zizzo *, Antonio Tamburello, Laura Castelnovo, Antonella Laria, Nicola Mumoli, \\ Paola Maria Faggioli, Ilario Stefani and Antonino Mazzone
}

Department of Internal Medicine, Azienda Socio Sanitaria Territoriale (ASST) Ovest Milanese, Milan, Italy

Acting on the cytokine cascade is key to preventing disease progression and death in hospitalised patients with COVID-19. Among anti-cytokine therapies, interleukin (IL)-6 inhibitors have been the most used and studied since the beginning of the pandemic. Going through previous observational studies, subsequent randomised controlled trials,

OPEN ACCESS

Edited by:

Alexei A. Grom,

Cincinnati Children's Research

Foundation, United States

Reviewed by:

Shetty Ravi Dyavar,

Adicet Bio, Inc, United States

Delia Goletti,

National Institute for Infectious

Diseases Lazzaro Spallanzani (IRCCS),

Italy

*Correspondence:

Gaetano Zizzo

gaetano.zizzo@asst-ovestmi.it

Specialty section:

This article was submitted to

Autoimmune and

Autoinflammatory Disorders,

a section of the journal

Frontiers in Immunology

Received: 14 October 2021

Accepted: 25 January 2022

Published: 22 February 2022

Citation:

Zizzo G, Tamburello A, Castelnovo L, Laria A, Mumoli N, Faggioli PM, Stefani I and Mazzone A (2022) Immunotherapy of COVID-19: Inside and Beyond IL-6 Signalling.

Front. Immunol. 13:795315.

doi: 10.3389/fimmu.2022.795315 and meta-analyses, we focused on the baseline characteristics of the patients recruited, identifying the most favourable features in the light of positive or negative study outcomes; taking into account the biological significance and predictivity of IL-6 and other biomarkers according to specific thresholds, we ultimately attempted to delineate precise windows for therapeutic intervention. By stimulating scavenger macrophages and T-cell responsivity, IL-6 seems protective against viral replication during asymptomatic infection; still protective on early tissue damage by modulating the release of granzymes and lymphokines in mild-moderate disease; importantly pathogenic in severe disease by inducing the proinflammatory activation of immune and endothelial cells (through transsignalling and trans-presentation); and again protective in critical disease by exerting homeostatic roles for tissue repair (through cis-signalling), while IL-1 still drives hyperinflammation. IL-6 inhibitors, particularly anti-IL-6R monoclonal antibodies (e.g., tocilizumab, sarilumab), are effective in severe disease, characterised by baseline IL-6 concentrations ranging from 35 to $90 \mathrm{ng} / \mathrm{mL}$ (reached in the circulation within 6 days of hospital admission), a ratio of partial pressure arterial oxygen (PaO2) and fraction of inspired oxygen (FiO2) between 100 and $200 \mathrm{mmHg}$, requirement of high-flow oxygen or non-invasive ventilation, C-reactive protein levels between 120 and $160 \mathrm{mg} / \mathrm{L}$, ferritin levels between 800 and $1600 \mathrm{ng} / \mathrm{mL}$, D-dimer levels between 750 and $3000 \mathrm{ng} / \mathrm{mL}$, and lactate dehydrogenase levels between 350 and 500 U/L. Granulocyte-macrophage colony-stimulating factor inhibitors might have similar windows of opportunity but different age preferences compared to IL-6 inhibitors (over or under 70 years old, respectively). Janus kinase inhibitors (e.g., baricitinib) may also be effective in moderate disease, whereas IL-1 inhibitors (e.g., anakinra) may also be effective in critical disease. Correct use of biologics based on therapeutic windows is essential for successful outcomes and could inform future new trials with more appropriate recruiting criteria.

Keywords: COVID-19, cytokines, IL-6, therapy, tocilizumab, sarilumab, baricitinib, anakinra 


\section{INTRODUCTION}

As of October 1, 2021, the Coronavirus Disease 2019 (COVID19) pandemic has caused over $200,000,000$ cases and more than $4,500,000$ deaths (1). Although severe acute respiratory syndrome coronavirus 2 (SARS-CoV-2) infection is more often asymptomatic, on the other hand healthy carriers importantly contribute to the spread of the virus, and COVID-19 can manifest itself in different forms of severity, namely mild, moderate, severe and critical.

Mild forms affect patients with respiratory symptoms who are generally not hospitalised and do not require supplemental oxygen. Moderate forms affect patients with viral pneumonia who require low-flow supplemental oxygen (LFO, $\leq 5$ liters per minute). Severe forms affect patients with bilateral interstitial pneumonia and acute respiratory distress syndrome (ARDS) requiring high-flow oxygen (HFO) or non-invasive ventilation (NIV). Critical forms affect patients admitted to intensive care unit (ICU) with severe ARDS, shock, and/or multiple organ failure, requiring invasive mechanical ventilation (IMV) with or without other organ support therapies, such as vasopressors, extracorporeal membrane oxygenation (ECMO), or dyalisis (2).

Severe and critical forms represent $14-15 \%$ and $2-5 \%$ of cases, respectively (3-6). Such life-threatening conditions are believed to result from a SARS-CoV-2-induced respiratory and systemic autoinflammatory disease, in which a dysregulated immune response, associated with exuberant cytokine release, would ultimately account for widespread vascular and tissue damage (7). Cytokines play a central role in the pathogenesis of COVID19 , emerging both as useful biomarkers in predicting disease evolution and as strategic targets for therapy. Discrete clusters of cytokines and chemokines are differentially expressed according to disease stage, with molecules involved in lymphoid priming being upregulated in moderate disease, and molecules involved in

Abbreviations: ACE2, angiotensin-converting enzyme 2; ARDS, acute respiratory distress syndrome; CAR-T, chimeric antigen receptor T-cell therapy; CCL, C-C motif chemokine ligand; $\mathrm{CD}$, cluster of differentiation; $\mathrm{CPAP}$, continuous positive airway pressure; CXCL, C-X-C motif chemokine ligand; COVID-19, Coronavirus Disease 2019; CRP, C-reactive protein; CRS, cytokine release syndrome; DAMPs, damage-associated molecular patterns; ECMO, extracorporeal membrane oxygenation; FABP4, fatty acid-binding protein 4; Fc, fragment crystallisable; FCN1, ficolin-1; G-CSF, granulocyte colony-stimulating factor; GM-CSF, granulocyte-macrophage colony-stimulating factor; gp130, 130-kDa glycoprotein; HFO, high-flow oxygen; i, inhibitors; HMGB1, high mobility group box-1; ICU, intensive care unit; IFN, interferon; IL, interleukin; IMV, invasive mechanical ventilation; JAK, Janus kinase; LDH, lactate dehydrogenase; LFO, low-flow oxygen; M1 or M2, type-I or type-II (classically or alternatively) activated macrophages; mAbs, monoclonal antibodies; MAS, macrophage activation syndrome; NETs, neutrophil extracellular traps; NIAID, National Institute of Allergy and Infectious Diseases; NIH, National Institutes of Health; NIV, noninvasive ventilation; NLRP3, NLR family pyrin domain containing 3; PAMPs, pathogen-associated molecular patterns; $\mathrm{PaO} 2 / \mathrm{FIO} 2$ ratio, pressure of arterial oxygen to fractional inspired oxygen concentration; -R, receptor; -RA, receptor antagonist; RCT, randomised controlled trial; RORC, retinoic acid orphan receptor $\mathrm{C}$; s-, soluble; $\mathrm{SaO} 2$, peripheral arterial oxygen saturation; SARS-CoV-2, severe acute respiratory syndrome coronavirus 2; SOCS, suppressor of cytokine signalling; SPP1, secreted phosphoprotein 1; STAT, signal transducer and activator of transcription; suPAR, soluble urokinase plasminogen activator receptor; TH, T helper; TH-GM, GM-CSF-producing T helper cells; TLR, Toll-like receptor; TNF, tumor necrosis factor; Treg, regulatory $\mathrm{T}$ cells. myeloid differentiation and migration being overexpressed in severe disease. A deep understanding of the qualitative, quantitative and temporal differences in cytokine pathways is therefore essential to appropriately choosing the right drug and the right timing for effective treatment $(7,8)$. While direct antiviral therapies may be useful in the early stages of the disease to inhibit virus replication, immunomodulatory and anti-cytokine therapies aimed at targeting harmful hyperinflammation would be crucial in the overt stages, to avoid critical deterioration and fatal outcomes. Since mortality rates can exceed $80 \%$ in critically-ill patients requiring $\operatorname{IMV}(9,10)$, acting in severe progressive forms appears to be of the utmost importance in reducing lethality.

\section{IL-6 IN COVID-19 INFLAMMATORY CASCADE: RATIONALE FOR A KEY ROLE IN SEVERE DISEASE PROGRESSION}

IL-6 levels in the circulation and bronchoalveolar lavage fluid of patients with COVID-19 progressively increase with disease severity $(11,12)$, reaching their peaks in critically-ill patients $(13,14)$. In the lungs of COVID-19 patients, IL- 6 can be released by SARS-CoV-2 infected respiratory epithelial cells $(15,16)$, as well as by infiltrating $\mathrm{CD} 14^{+} \mathrm{CD} 16^{+}$monocytes-macrophages and $\mathrm{CD} 4^{+} \mathrm{T}$ cells (17).

IL-6 can substantially contribute to the dysregulation of the immune response in COVID-19, basically by acting in two directions: on one side, it may cause a dysfunction of natural killer and cytotoxic $\mathrm{CD}^{+} \mathrm{T}$ cells associated with perforin and granzyme down-regulation (18), thereby dampening antiviral defenses; on the other side, it may inhibit the differentiation of regulatory $\mathrm{T}$ cells and elicit a Thelper 17 (TH17)-like polarization of $\gamma / \delta$ and $\alpha / \beta \mathrm{CD}^{+} \mathrm{T}$ cells, thus leading to uncontrolled hyperinflammation (7). At advanced stages, these mechanisms would result into a macrophage activation syndrome (MAS)-like condition, characterised by lymphocyte exhaustion and aberrant innate immune responses, vascular leakage, exudative-phase ARDS, coagulopathy, and multi-organ failure $(7,19)$. In severe COVID-19, in fact, increased levels of IL-6 have been found to be associated with higher viral load (20), lymphopenia and neutrophilia $(11,21)$, systemic inflammation (22), hypoxemia (23), and poor prognosis $(22,23)$. On the other hand, certain polymorphisms in the IL- 6 receptor gene that attenuate IL-6 signalling have been shown to be protective against disease progression, resulting in a lower risk of hospitalisation for COVID-19 (24). Therapeutic blockade of IL-6 may therefore represent an effective strategy to prevent worsening of respiratory status and reduce overall mortality in these patients.

\section{INHIBITORS OF IL-6 SIGNAL IN COVID-19: WHAT TO HIT AND WHEN?}

IL-6 is a pleiotropic cytokine with multiple functions and a complex signalling involving two receptor subunits: IL-6R $\alpha$ (IL$6 \mathrm{R})$ and IL-6R $\beta$ (130-kDa glycoprotein or gp130). Several 
signalling modalities have been identified, namely: cis-signalling (or classic mode), in which IL-6 binds to membrane IL-6R (e.g., on macrophages, hepatocytes, megacaryocytes, intestinal epithelial cells) and gp130; trans-signalling, in which a complex formed by IL-6 and soluble IL-6R (mainly cleaved by a disintegrin and metalloprotease-17 or ADAM-17) binds to membrane gp130 (e.g., in endothelial cells, neutrophils, monocytes, pneumocytes); and trans-presentation, in which IL-6 first binds to IL-6R on the membrane of one cell (e.g., dendritic cells) and then the complex binds to gp130 in another cell (e.g., CD4 ${ }^{+} \mathrm{T}$ cells) (25-27). Different modes result in different effects: cis-signalling mediates host protection against intracellular pathogens and tissue homeostasis, by upregulating opsonins in the liver and by inducing scavenger and regulatory functions in macrophages; trans-signalling drives proinflammatory activation of pneumocytes, adipose tissueassociated macrophages, gut-associated immune cells, neutrophils and endothelial cells; while trans-presentation promotes T-cell differentiation into pathogenic TH17 cells $(25,26)$ (Figure 1).

IL-6 trans-signalling and trans-presentation are most likely pathogenic in severe progressive COVID-19: through transpresentation, IL-6 may act, via signal transducer and activator of transcription (STAT) 3, to polarize granulocyte-macrophage colony-

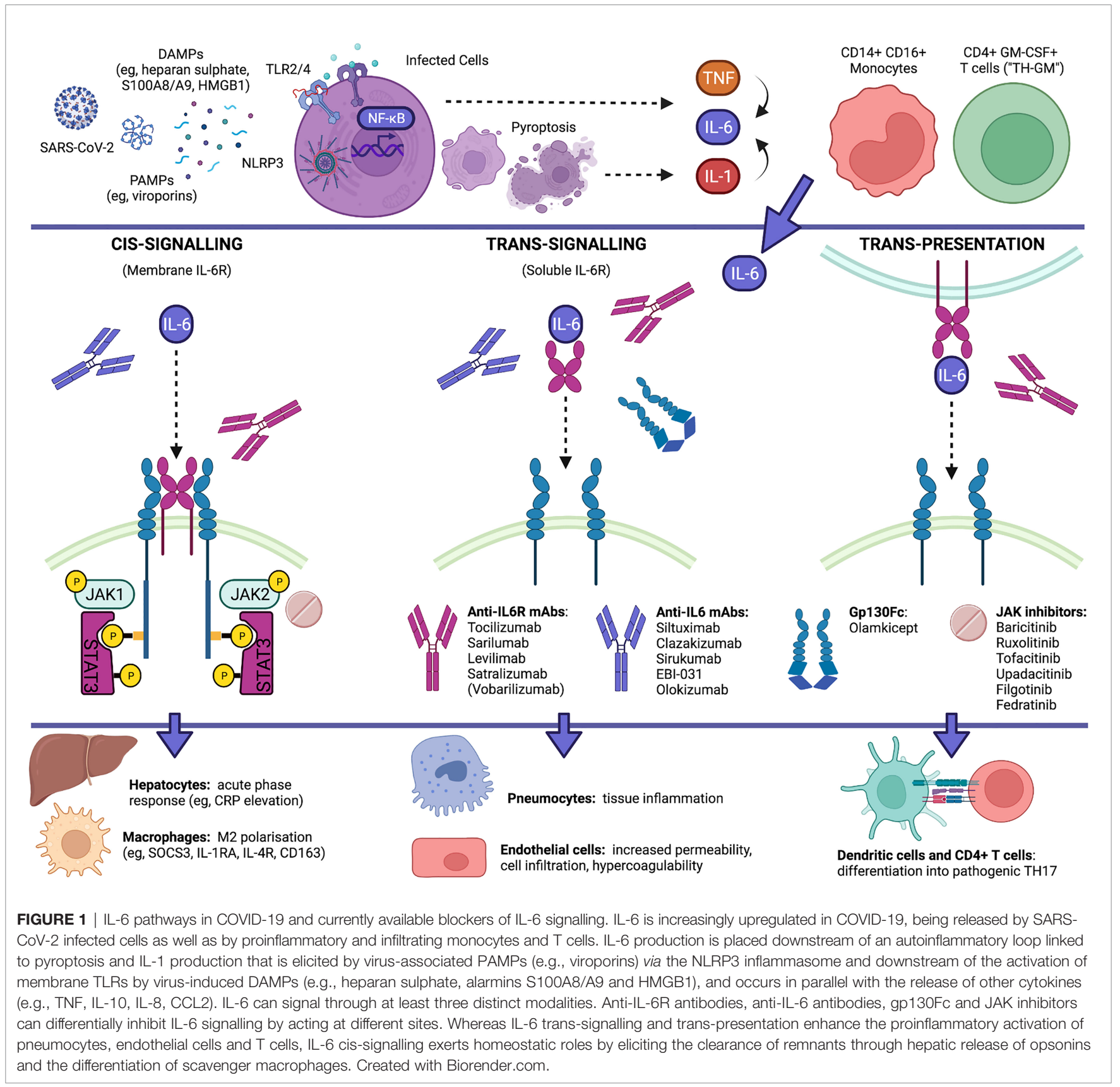


stimulating factor (GM-CSF)-producing T cells (“TH-GM"), induced by IL-7, IL-23 and IL-1, into full-blown TH17 cells, thus amplifying neutrophil recruitment and activation (7); through transsignalling, IL-6 may further contribute to upregulate chemokines (e.g., CCL2, IL-8), adhesion molecules (e.g., intercellular adhesion molecule-1, while internalizing vascular endothelial cadherin), their ligands (e.g., CD11b/CD18 integrin), and procoagulant factors (e.g., tissue factor-1, factor-III), thereby leading to increased endothelial permeability, $\mathrm{CD} 14^{+} \mathrm{CD} 16^{+}$(intermediate/non-classical) monocyte and neutrophil migration from peripheral blood into inflamed tissues, formation of neutrophil extracellular traps (NETs), neutrophil-endothelial cell interactions, and hypercoagulability ( 7 , $12,17,28-30)$. IL-6 trans-signalling and trans-presentation may therefore account for diffuse inflammation at various levels, including pulmonary, vascular, intestinal, and obesity-enhanced inflammation, up to the condition of shock, secondary to cytokine-mediated multi-organ dysfunction, vascular leakage and microthrombosis.

Whereas the soluble decoy receptor sgp130Fc (e.g., olamkicept) selectively blocks trans-signalling, anti-IL-6 monoclonal antibodies (e.g., siltuximab, clazakizumab, olokizumab) can block cis-signalling and trans-signalling, and anti-IL-6R monoclonal antibodies (e.g., tocilizumab, sarilumab) can block all three mechanisms, including trans-presentation (25). By acting on soluble IL-6R, monoclonal antibodies might also affect the signalling of IL-27p28/IL-30, possibly interfering with the induction of CXCL10 (31), another chemokine that is strongly upregulated in COVID-19 (7). Since tocilizumab, but not siltuximab, has been shown from meta-analyses to be effective in patients hospitalised with COVID-19 (32), blocking transpresentation and IL-6-induced TH17 polarization would be particularly crucial to prevent severe to critical disease progression.

Conversely, IL-6 cis-signalling mainly drives hepatic synthesis and secretion of acute-phase reactants (e.g., CRP elevation in the blood), and exerts negative feedback mechanisms on proinflammatory cytokines, by suppressing their production (e.g., TNF) (33), stimulating their decoy receptors (e.g., sTNFRp55, IL1RA) (34), or inducing negative regulators of their intracellular pathways (e.g., suppressor of cytokine signalling 3, or SOCS3, inhibiting IL-6, IL-7 and IL-23 signals; and IL-4R, inhibiting TH17 maturation) $(7,25,26,35)$. In the circulation of patients with COVID-19, IL-6 reaches its peak at advanced stages, on average after the second week of disease, and, notably, this increase is accompanied by peak concentrations of IL-10 and CRP $(11,13)$. This suggests that in critical COVID-19, strong elevation of IL-6 (e.g., > 100-120 pg/mL) and CRP levels (e.g., > $160-200 \mathrm{mg} / \mathrm{L}$ ) could reflect augmented IL-6 cis-signalling in the attempt to exert homeostatic roles, while IL-10 further strengthens the predominance of SOCS3 over STAT3 signal (36). Therefore, inhibiting membrane IL-6R at this stage would not be useful (and might even worsen inflammation), as indicated by poor outcomes with tocilizumab in critically-ill patients. Furthermore, since IL-6 cis-signalling elicits host defence against pathogens and promotes the growth and survival of hepatocytes, megacaryocytes and intestinal epithelial cells $(25,26)$, membrane IL-6R blockade might promote adverse events and complications, including serious infections (e.g., ventilator-associated pneumonia) $(37,38)$, increased transaminases, thrombocytopenia and intestinal perforation, particularly in patients who are critically-ill with COVID-19, as they are intubated, immunosuppressed, and inflamed or hypoperfused at multiple levels.

In early stages of COVID-19, in which IL-6 may play protective roles, IL-6R blockade would not be useful either. Many viruses, in fact, including SARS-CoV-2, are able to induce SOCS3 as a virulence factor, which inhibits STAT1/IFN and gp130/JAK2/ STAT3 signals to elude antiviral responses and virus clearance, thus allowing viral replication (39-41); collaterally, STAT3 downmodulation results in a compensatory induction of IL-6 mediated by nuclear factor kappa-light-chain-enhancer of activated B cells (NF-kB) (41), thereby already mimicking the effects of anti-IL-6R agents. Moreover, viral pneumonia in mild-moderate COVID-19 might be substantially mediated by cytokines and cellular pathways pathogenically upstream of IL-6, such as IL-33, IL-9 or IFN $\gamma$, which can be released by virus-damaged respiratory epithelial cells, type- 2 innate lymphoid cells and $\gamma / \delta$ T cells (7); indeed, at this stage, IL-6 may negatively modulate T-cell release of IFN $\gamma$ and granzymes $(18,42)$.

In fact, tocilizumab was observed to reduce IL-17 and TNF (i.e., cytokines associated with severe disease), yet it provoked a transitory increase in IFN $\gamma$ (probably associated with moderate disease), and a strong increase in IL-6 and IL-10 levels (highly associated with critical disease) (43).

Taken together, benefits from therapeutic blockade of IL- 6 can be obtained in COVID-19 by disrupting proinflammatory IL- 6 transpresentation and trans-signalling, which would be predominant and pathogenic in severe disease; anti-IL-6 treatment should therefore be given to patients with severe and rapidly progressive COVID-19, within the second week of symptom onset (or within the first week of hospitalisation), that is before the increased IL-6 and CRP levels further rise uncontrollably. By contrast, IL-6 inhibition no longer appears to be useful in critically-ill patients, in whom IL-6 cissignalling predominates and would now exert anti-inflammatory and homeostatic roles. IL-6 inhibition does not seem to be useful even in early stages of COVID-19, as IL-6 signalling would be protective, indeed already blocked by the virus, and mild-moderate disease would be primarily driven by viral replication and additional upstream cytokines (Figure 2).

\section{TOCILIZUMAB IN EARLY OBSERVATIONAL STUDIES: WHAT HAVE WE LEARNED FROM THE ITALIAN EXPERIENCE?}

Tocilizumab is an anti-IL-6R monoclonal antibody that is approved, and widely available in both intravenous and subcutaneous formulations, for certain acute and chronic inflammatory disorders that share many clinical and pathogenic aspects with severe COVID-19, such as autoinflammatory febrile diseases (e.g., Still's disease), autoimmune diseases that are complicated by interstitial lung disease (e.g., rheumatoid 


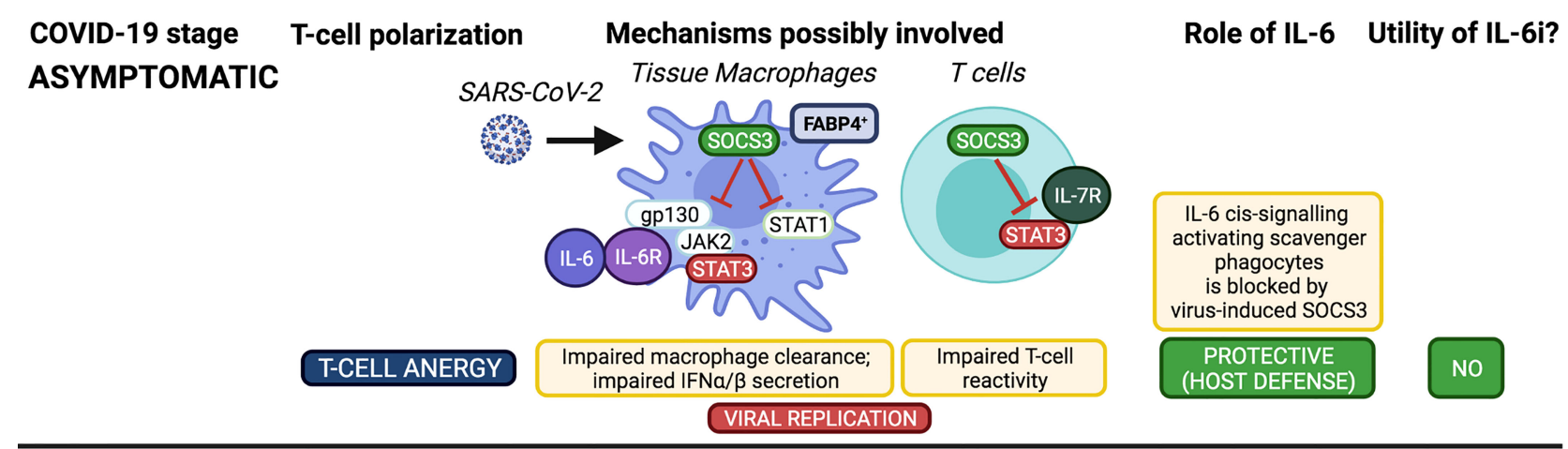

MILD-MODERATE

Infected Pneumocytes Innate Lymphoid Cells (ILC2)
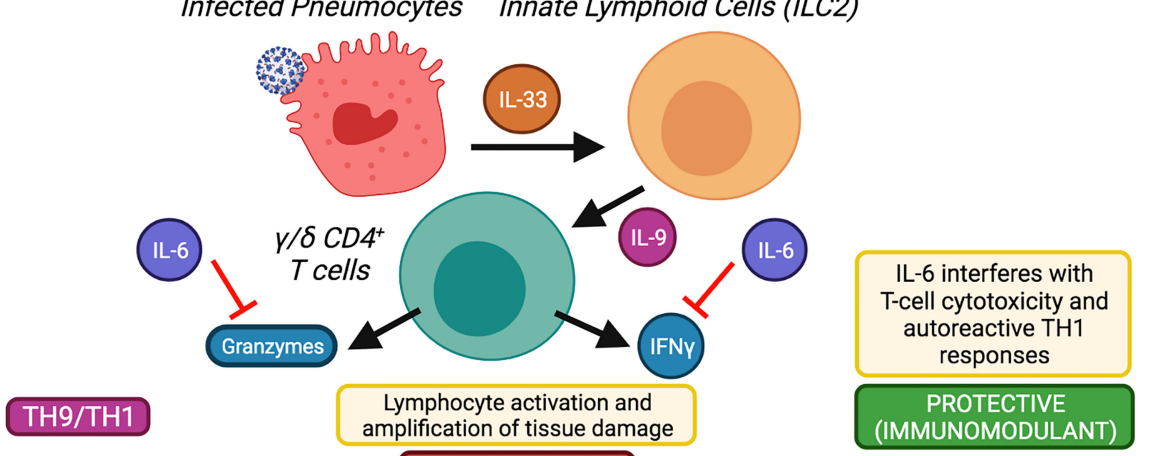

\section{SEVERE} VIRAL PNEUMONIA

IL-6 trans-signalling and trans-presentation stimulate TH17 and aberrant innate immune responses

PATHOGENIC (PROINFLAMMATORY)

\section{CRITICAL}

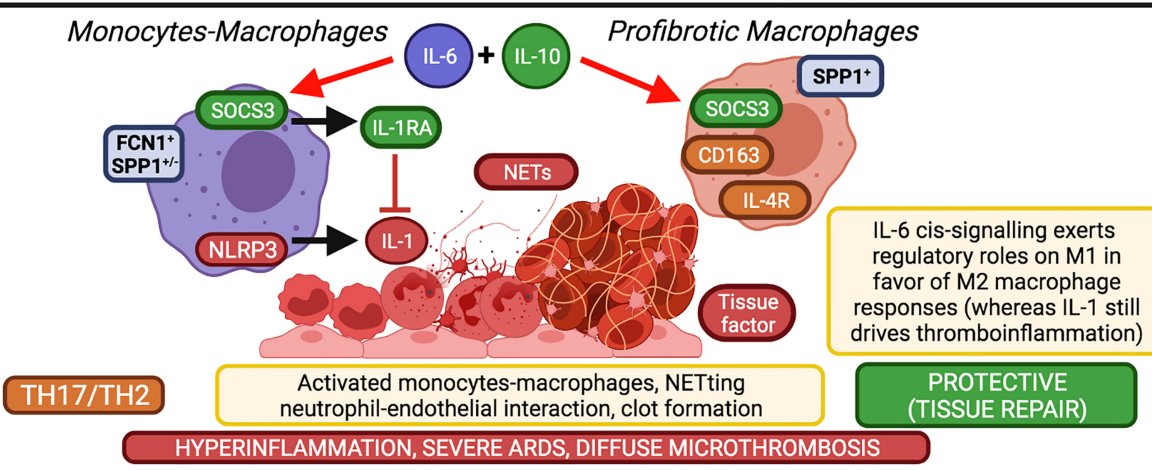

FIGURE 2 | Implications of IL-6 in COVID-19 pathogenesis and therapeutic opportunity of using IL-6 inhibitors according to disease stage. Taking into account the well-established functions of IL-6 on immune cells (i.e. pro-M2, anti-Treg, anti-cytotoxic, anti-TH1, pro-TH17), here we schematically summarize the differential roles of IL-6 in the inflammatory cascade of COVID-19 according to disease stages and supposed T-cell polarization, which affect the usefulness or otherwise of IL-6 inhibitors (IL-6i). Created with Biorender.com. 
arthritis), vasculitis (e.g., giant cell arteritis), and cytokine storm syndromes (e.g., MAS complicating Still's disease, chimeric antigen receptor $\mathrm{T}$-cell therapy-induced cytokine release syndrome or CAR-T cell-induced CRS). In these contexts, tocilizumab can efficiently act on fever, fatigue, pain, anxiety and depression, anemia, acute-phase response, deterioration of lung function, and vascular manifestations (44).

Italy was among the first countries after China to face the COVID-19 outbreak. It was soon realised that patients hospitalised with COVID-19 who worsened and died had developed a hyperinflammatory reaction largely responsible for severe ARDS and multi-organ failure. Tocilizumab and other IL-6 inhibitors (i.e., sarilumab, siltuximab) were then tried as compassionate off-label use in an effort to relieve harmful inflammation.

From the literature, we identified 10 retrospective cohort studies, published between May 9, 2020, and January 8, 2021, that enrolled patients hospitalised with COVID-19 during the "first wave" of the outbreak in Italy (overall between the end of February and the end of May, 2020), who underwent treatment with IL-6 inhibitors versus standard of care only (45-54). Studies were somewhat heterogenous in the number of patients recruited or included in the treatment arm, as well as in treatment protocols (including route of administration, dosage, number and timing of infusions), co-treatments, and disease severity ( $\mathrm{PaO} 2 / \mathrm{FIO} 2$ ratio and magnitude of systemic inflammation) (Table $\mathbf{1}$ ).

It was noteworthy that most of the studies with positive results had recruited at least 50 patients in the treatment arm (46, $50,52,54)$, whereas those showing negative or non-significant results included fewer patients $(45,47,48,51)$, thus suggesting that results might have been influenced by the small size of the cohorts. Most of the patients were men ( $\geq 70 \%$ ), aged between 59 and 64 years old; however, some studies with negative or partial results included more women (53) and/or patients that were on average older than 70 (53) or younger than 59 (51). No obvious difference in the overall distribution of comorbidities (e.g., hypertension, diabetes, heart disease) was noticed between studies with positive and negative results.

According to the data available, patients were generally enrolled for treatment within the second week of symptom onset and within 4 days of hospitalisation. Tocilizumab was given mainly intravenously, at various doses (400-800 mg), in single or multiple infusions (1 to 3 infusions, at intervals of 12-72 hours). It was noted that two infusions could increase the rate of secondary infections and bacteremia compared to a single infusion $(48,50)$, while the effects of subcutaneous administration on mortality and composite outcomes (i.e., IMV + death) lost statistical significance in the subgroup analysis (50).

Concerning concomitant therapies, standard of care originally included drugs (e.g., hydroxychloroquine, azithromycin, lopinavir/ ritonavir) subsequently proved ineffective against COVID-19 (55, 56), while remdesivir was not available or administered to a negligible number of patients $(47,52)$. Since the RECOVERY data on the efficacy of intermediate-to-low doses of dexamethasone and the benefit of combining tocilizumab with dexamethasone on overall survival were published later $(57,58)$, standard of care and treatment protocols did not yet include the use of glucocorticoids. In some cases, steroids were given to a minority of patients $(<50 \%)(47$, 50,53 ), generally at high doses (i.e., methylprednisolone $1-2 \mathrm{mg} / \mathrm{Kg}$ per day) as supportive therapy $(45,53)$. In one study, in which all tocilizumab patients were co-treated with lower doses of steroids (i.e., methylprednisolone $0.5-1 \mathrm{mg} / \mathrm{Kg}$ per day, tapered and suspended within 7-10 days), tocilizumab showed good results (54).

Most importantly, patients who successfully responded to IL6 inhibitors had severe disease, but neither critical nor moderate disease. Studies in which most of the patients required IMV showed negative results (47), whereas treatment was effective in case series without or with low numbers of intubated patients $(49,50,54)$. On the other hand, due to the overwhelming of ICU during the first months of the outbreak, patients that underwent IMV were probably less than they should have been $(48,51)$. Parameters to be considered more reliable of disease severity could instead be the pressure of arterial oxygen to fractional inspired oxygen concentration $(\mathrm{PaO} 2 / \mathrm{FIO} 2)$ ratio and possibly some inflammatory markers. In fact, patients from studies reporting significantly reduced mortality with IL-6 inhibitors had baseline median $\mathrm{PaO} 2 / \mathrm{FIO} 2$ ratios between 110 and 200 $\mathrm{mmHg}$ (i.e., severe disease) $(46,49,50,52)$; studies with median $\mathrm{PaO} 2 / \mathrm{FIO} 2$ ratios between 100 and $110 \mathrm{mmHg}$ (i.e., severe-tocritical disease) showed numerically but not statistically significant differences in mortality $(48,53)$ and in some cases reported a significant reduction in IMV requirement (53); whereas studies with median $\mathrm{PaO} 2 / \mathrm{FIO} 2$ ratios $<100$ (i.e., critical disease) or $>200 \mathrm{mmHg}$ (i.e., moderate disease) failed to show substantial differences in mortality, IMV requirement or hospital discharge $(45,47,51)$.

Looking at inflammatory markers, studies with positive results reported median CRP values around 120-130 mg/L (46, $49)$, whereas studies with only partial results or negative results reported values $\geq 160 \mathrm{mg} / \mathrm{L}(48,53)$ or $\leq 80 \mathrm{mg} / \mathrm{L}(45,47)$. Moreover, patient series with ferritin levels $<1600 \mathrm{ng} / \mathrm{mL}$ and $\mathrm{D}$ dimer levels < $3000 \mathrm{ng} / \mathrm{mL}(50,54)$ showed better outcomes compared to those with higher values $(51,53)$ (Table 1).

Univariate and multivariate analyses confirmed many of these observations. Compared to patients who did not respond to IL-6 inhibitors, patients who improved after treatment showed significant differences in baseline median values of: age (59-63 versus 74 years old) $(48,59)$, male percentage (100 versus $70 \%)$ (48), $\mathrm{PaO} 2 / \mathrm{FIO} 2$ ratio $(112-137$ versus $81-86 \mathrm{mmHg})(48,51)$, CRP (128 versus $186 \mathrm{mg} / \mathrm{L})$ (48), IL-6 (52-58 versus 99-120 pg/ $\mathrm{ml})(51,59)$, and neutrophil-to-lymphocyte ratio (3.5 versus 8 ) (59). Similar numerical differences in age, $\mathrm{PaO} 2 / \mathrm{FIO} 2$ ratio, IL-6 and neutrophil-to-lymphocyte ratio were actually observed between ICU patients requiring IMV and non-ICU patients requiring NIV/HFO $(47,59)$, further indicating that IL-6 blockade responders belong to this second category of patients.

At multivariate regression analysis, independent predictors of clinical improvement and survival upon treatment were: age $\leq$ $70-75$ years old $(48,59,60), \mathrm{PaO} 2 / \mathrm{FIO} 2$ ratio $\geq 100(48,51,60)$, IL-6 values $<91 \mathrm{pg} / \mathrm{mL}(59)$, administration of tocilizumab within 6 days (61), and no need of IMV (60). Conversely, independent predictors of ICU admission and mortality were: comorbidities (in particular kidney impairment) (53), IMV at 
TABLE 1 | Early Italian observational cohort studies with IL-6 inhibitors in COVID-19,

\begin{tabular}{|c|c|c|c|c|c|c|c|c|c|c|}
\hline $\begin{array}{l}\text { PROVED } \\
\text { EFFICACY }\end{array}$ & NO & YES & NO & PARTIAL & YES & YES & NO & YES & PARTIAL & YES \\
\hline $\begin{array}{l}\text { Retrospective Cohort } \\
\text { Study } \\
\text { [Ref.] }\end{array}$ & $\begin{array}{l}\text { Colaneri } \\
\text { et al. } \\
\text { (45) }\end{array}$ & $\begin{array}{l}\text { Capra } \\
\text { et al. } \\
\text { (46) }\end{array}$ & $\begin{array}{l}\text { Quartuccio } \\
\text { et al. } \\
\text { (47) }\end{array}$ & $\begin{array}{l}\text { Campochiaro } \\
\text { et al. } \\
(48)\end{array}$ & $\begin{array}{l}\text { Gritti } \\
\text { et al. } \\
(49)\end{array}$ & $\begin{array}{l}\text { Guaraldi } \\
\text { et al. } \\
\text { (50) }\end{array}$ & $\begin{array}{l}\text { Della Torre } \\
\text { et al. } \\
\text { (51) }\end{array}$ & $\begin{array}{l}\text { Rossotti } \\
\text { et al. } \\
(52)\end{array}$ & $\begin{array}{l}\text { Canziani } \\
\text { et al. } \\
\text { (53) }\end{array}$ & $\begin{array}{l}\text { Castelnovo } \\
\text { et al. } \\
\text { (54) }\end{array}$ \\
\hline \multicolumn{11}{|c|}{ STUDY CHARACTERISTICS: } \\
\hline Date of publication & $\begin{array}{l}\text { May 9, } \\
2020\end{array}$ & $\begin{array}{l}\text { May 13, } \\
2020\end{array}$ & $\begin{array}{l}\text { May } 15 \\
2020\end{array}$ & $\begin{array}{l}\text { May 22, } \\
2020\end{array}$ & $\begin{array}{l}\text { Jun } 20 \\
2020\end{array}$ & $\begin{array}{l}\text { Jun } 24 \\
2020\end{array}$ & $\begin{array}{l}\text { Jul 3, } \\
2020\end{array}$ & $\begin{array}{l}\text { Jul } 8 \\
2020\end{array}$ & $\begin{array}{l}\text { Jul } 8 \\
2020\end{array}$ & $\begin{array}{l}\text { Jan } 8 \\
2021\end{array}$ \\
\hline $\begin{array}{l}\text { Period of enrollment } \\
\text { (2020) }\end{array}$ & $\begin{array}{l}\text { Mar } 14- \\
\text { Mar } 27\end{array}$ & $\begin{array}{l}\text { Feb } 26- \\
\text { Apr } 2\end{array}$ & $\begin{array}{l}\text { Feb } 29- \\
\text { Apr } 6\end{array}$ & $\begin{array}{l}?- \\
\text { Mar } 19\end{array}$ & $\begin{array}{l}\text { Feb } 23- \\
\text { Apr } 9\end{array}$ & $\begin{array}{l}\text { Feb } 21- \\
\text { Apr } 30\end{array}$ & $\begin{array}{l}\text { Mar } 14- \\
\text { Apr } 2\end{array}$ & $\begin{array}{l}\text { Mar } 13- \\
\text { Apr } 3\end{array}$ & $\begin{array}{l}\text { Mar } 15- \\
\text { Apr } 22\end{array}$ & $\begin{array}{l}\text { Mar } 6- \\
\text { May } 30\end{array}$ \\
\hline $\begin{array}{l}\text { Treatment arm } \\
\text { (route, dosage, } \\
\text { number of doses) }\end{array}$ & $\begin{array}{l}\text { Tocilizumab } \\
\text { IV } 8 \mathrm{mg} / \mathrm{kg} \\
\text { (x2) }\end{array}$ & $\begin{array}{l}\text { Tocilizumab } \\
\text { IV } 400 \mathrm{mg} \text { or } \\
\text { SC } 324 \mathrm{mg} \\
\text { (x1) }\end{array}$ & $\begin{array}{l}\text { Tocilizumab } \\
\text { IV } 8 \text { mg/Kg } \\
\text { (x1) }\end{array}$ & $\begin{array}{l}\text { Tocilizumab } \\
\text { IV } 400 \mathrm{mg} \\
(x 1-2)\end{array}$ & $\begin{array}{l}\text { Siltuximab } \\
\text { IV } 11 \mathrm{mg} / \\
\mathrm{kg} \\
(\mathrm{x} 1-2)\end{array}$ & $\begin{array}{l}\text { Tocilizumab } \\
\text { IV } 8 \text { mg/kg (x2) } \\
\text { or SC } 324 \text { mg (x1) }\end{array}$ & $\begin{array}{l}\text { Sarilumab } \\
\text { IV } 400 \mathrm{mg} \\
\text { (x1) }\end{array}$ & $\begin{array}{l}\text { Tocilizumab } \\
\text { IV } 8 \mathrm{mg} / \mathrm{kg} \\
(x 1-2)\end{array}$ & $\begin{array}{l}\text { Tocilizumab } \\
\text { IV } 8 \mathrm{mg} / \mathrm{Kg} \\
\text { (x2) }\end{array}$ & $\begin{array}{l}\text { Tocilizumab } \\
\text { IV } 8 \mathrm{mg} / \mathrm{kg} \\
\text { (x2-3) }\end{array}$ \\
\hline $\begin{array}{l}\text { Number of patients } \\
\text { recruited }\end{array}$ & 112 & 85 & 111 & 65 & $\begin{array}{l}60 \\
\text { matched } \\
\text { (218 total) }\end{array}$ & 544 & 56 & 222 & 128 & 112 \\
\hline $\begin{array}{l}\text { Number of patients } \\
\text { in the treatment arm }\end{array}$ & 21 & 62 & 42 & 32 & 30 & $\begin{array}{l}179 \\
(88 \mathrm{iv}+91 \mathrm{sc})\end{array}$ & 28 & 74 & 64 & 50 \\
\hline \multicolumn{11}{|c|}{ PATIENT CHARACTERISTICS (treatment arm): } \\
\hline (Median) Age (years old) & 62 & 63 & 62 & 64 & 64 & 64 & 56 & 59 & (mean) 73 & (mean) 61 \\
\hline Male sex (\%) & 90.5 & 73 & 79 & 91 & 77 & 71 & 85 & 82 & 63 & 70 \\
\hline $\begin{array}{l}\text { (Median) Number of Days } \\
\text { from hospitalization }\end{array}$ & 2 & $\leq 4$ & & & & & 2 & & & 4 \\
\hline $\begin{array}{l}\text { (Median) Number of Days } \\
\text { from symptom onset }\end{array}$ & & $\leq 11$ & & 11 & & 7 & 7 & & 13 & \\
\hline Hypertension (\%) & 38 & 46 & 48 & 37 & 40 & 45 & 21 & & 52 & 34 \\
\hline Type-2 Diabetes (\%) & 9.5 & 14 & & 12 & 20 & 13 & 11 & & & 6 \\
\hline Heart disease (\%) & 9.5 & 14 & & 18 & 13 & 12 & 8 & & & \\
\hline $\begin{array}{l}\text { Glucocorticoids (\%) } \\
\text { (Methylprednisolone dose) }\end{array}$ & $\begin{array}{l}100 \\
\text { (MP } 1 \\
\mathrm{mg} / \mathrm{Kg} \text { ) }\end{array}$ & 0 & $\begin{array}{l}38 \\
\text { (MP } 1 \\
\mathrm{mg} / \mathrm{Kg} \text { ) }\end{array}$ & 0 & 0 & 30 & 0 & & $\begin{array}{l}48 \\
\text { (MP 2-3 } \\
\mathrm{mg} / \mathrm{Kg} \text { ) }\end{array}$ & $\begin{array}{l}100 \\
\text { (MP 0.5-1 } \\
\mathrm{mg} / \mathrm{kg} \text { ) }\end{array}$ \\
\hline Remdesivir (\%) & & & $\leq 7$ & & & & & 9.5 & 0 & 0 \\
\hline Ambient air (\%) & & & & 0 & 0 & & 0 & & 0 & 0 \\
\hline LFO (\%) & & & & 0 & 0 & & 0 & & 8 & 4 \\
\hline HFO or NIV (\%) & & & & 100 & 83 & & 100 & & 72 & 96 \\
\hline IMV (\%) & & & 62 & 0 & 17 & 18 & 0 & & 20 & 0 \\
\hline $\begin{array}{l}\text { (Median) PaO2/FIO2 ratio } \\
\text { (mmHg) }\end{array}$ & 225 & $(\leq 300)$ & & 107 & 109 & 169 & $<100$ in $60 \%$ & 180 & (mean) 104 & \\
\hline (Median) CRP (mg/L) & 21 & 123 & 79 & 156 & 130 & 34-61 (partial data) & 143 & & (mean) 198 & \\
\hline (Median) Ferritin (ng/mL) & & & & 1400 & & 1168 (partial data) & 1849 & & (mean) 1638 & (mean) 1592 \\
\hline (Median) D-dimer (ng/mL) & & & 835 & & & $\begin{array}{l}\text { 1000-1210 (partial } \\
\text { data) }\end{array}$ & 1270 & & (mean) 3801 & (mean) 2931 \\
\hline (Median) LDH (U/L) & 445 & & 625 & 469 & 505.5 & 600-676 (partial data) & 468 & & (mean) 524 & \\
\hline \multicolumn{11}{|c|}{ OUTCOMES (treatment vs. standard-of-care): } \\
\hline (28-day) Clinical status & & & & 69 vs. 61 & & & 17 vs. 18 & & & (7-day CPAP) \\
\hline Improvement (\%) & & & & $(p=N S)$ & & & & & & 8 vs. 87 \\
\hline (28-day) Discharge (\%) & & 92 vs. 42 & $\begin{array}{l}\text { (35-day) } \\
24 \text { vs. } 100\end{array}$ & $\begin{array}{l}63 \text { vs. } 49 \\
\text { (p=NS) }\end{array}$ & & & 17 vs. 17 & & & $\begin{array}{l}\text { (overall) } \\
88 \text { vs. } 64.5\end{array}$ \\
\hline
\end{tabular}


TABLE 1 | Continued

\begin{tabular}{|c|c|c|c|c|c|c|c|c|c|c|}
\hline $\begin{array}{l}\text { PROVED } \\
\text { EFFICACY }\end{array}$ & NO & YES & NO & PARTIAL & YES & YES & NO & YES & PARTIAL & YES \\
\hline (28-day) IMV (\%) & (7-day ICU) & & & 13 vs. 6 & [HR & (14-day) & 6 vs. 7 & & (30-day) & \\
\hline [HR] & 14 vs. 13 & & & $(p=N S)$ & $\begin{array}{l}0.615 \\
p=0.072]\end{array}$ & 18 vs. 16 & & & $\begin{array}{l}17 \text { vs. } 48 \\
\text { ( } p=0.001) \\
{[H R 0.36,} \\
p=0.017]\end{array}$ & \\
\hline $\begin{array}{l}\text { (28-day) IMV or death } \\
\text { (\%) [HR] }\end{array}$ & & & & & & $\begin{array}{l}\text { [Adj HR 0.61, } \\
\mathrm{p}=0.020 \text { ] } \\
\text { (For } P / F \leq 150 \text { : } \\
\text { Adj HR } 0.19 \text {, } \\
\mathrm{p}=0.011 ; \\
\text { significance kept } \\
\text { for IV } \\
\text { administration only: } \\
\text { Adj HR } 0.55 \text {, } \\
\mathrm{p}=0.042 \text { ) }\end{array}$ & & 35 vs.? & & \\
\hline (28-day) Mortality (\%) & (7-day) & 8 vs. 58 & (35-day) & 16 vs. 33 & (30-day) & (14-day) & 2 vs. 5 & [HR 0.50, & (30-day) & (overall) \\
\hline [HR] & $\begin{array}{l}24 \text { vs. } 21 \\
\text { [OR } 0.78, \\
p=N S \text { ] }\end{array}$ & $\begin{array}{l}\text { [HR 0.035, } \\
p=0.004]\end{array}$ & 9.5 vs. 0 & $(p=N S)$ & $\begin{array}{l}33 \text { vs. } 53 \\
\text { [HR } 0.46, \\
p=0.040]\end{array}$ & $\begin{array}{l}7 \text { vs. } 20 \text { ( } p=0.0007) \\
\text { [Adj HR } 0.38 \text {, } \\
p=0.015] \\
\text { (Significance kept } \\
\text { for IV } \\
\text { administration only: } \\
\text { Adj HR } 0.29 \text {, } \\
p=0.048 \text { ) }\end{array}$ & $(p=N S)$ & $\mathrm{p}=0.035]$ & $\begin{array}{l}27 \text { vs. } 38 \\
\text { [HR } 0.61, \\
p=N S \text { ] }\end{array}$ & 12 vs. 34 \\
\hline Infections (\%) & & 0 vs. 0 & 65 vs. 0 & $\begin{array}{l}13 \text { vs. } 12 \\
\text { ( } 6 \% \text { one dose vs. } \\
33 \% \text { two doses, } \\
p=0.06 \text { ) }\end{array}$ & 43 vs.? & $\begin{array}{l}13 \text { vs. } 4 \\
(p<0.0001)\end{array}$ & 21 vs. 18 & 32 vs.? & 31 vs. 39 & 0 \\
\hline
\end{tabular}

LEGEND OF COLOURS: Green: numbers falling within the thresholds herein suggested (optimal study and patient characteristics), in relation with statistically significant results and substantial benefit on overall mortality (i.e., positive outcomes). Blue: numbers falling borderline (suboptimal study and patient characteristics), in relation with numerically but not statistically significant results, and substantial benefit on clinical status (e.g., IMV requirement, hospital discharge) (i.e., partially positive outcomes). Red: numbers falling outside of the thresholds (non-optimal study and patient characteristics), in relation with non-significant results, and no substantial benefit on either clinical status or overall mortality (i.e., negative outcomes).

Adj, adjusted; HR, hazard ratio; NS, not significant. 
baseline $(9,53)$, and D-dimer levels > $3500 \mathrm{ng} / \mathrm{ml}$ (61). Both tocilizumab and glucocorticoid use were predictors of reduced mortality at 14 days, in the case of tocilizumab specifically in patients not requiring IMV (62).

After treatment, good responders showed control values of ferritin returning $<1400 \mathrm{ng} / \mathrm{ml}$, D-dimer $<3000 \mathrm{ng} / \mathrm{ml}$ and lactate dehydrogenase $(\mathrm{LDH})<400 \mathrm{U} / \mathrm{L}$, while non-responders had ferritin $>1800 \mathrm{ng} / \mathrm{ml}$, D-dimer $>5000 \mathrm{ng} / \mathrm{ml}$ and $\mathrm{LDH}>500$ U/L (63). Lower levels of IL-6 24 hours after the infusion were found predictive of clinical improvement at day 7 (62). Irrespective of the main outcome, IL-6 inhibitors led to a fast decrease and normalisation of CRP levels, and a rapid resolution of fever and fatigue $(45,50,51,54,63)$.

\section{MORE CONCLUSIVE EVIDENCE: RESULTS FROM RAMDOMISED CONTROLLED TRIALS}

From the early stages of the pandemic, IL- 6 has been a welldocumented biomarker of disease severity and poor prognosis, and considered among the most promising targets in the treatment of the COVID-19 cytokine storm (19). About half of the clinical trials being conducted worldwide testing monoclonal antibodies for the management of severe COVID-19 have been performed with IL-6 inhibitors, and in particular with tocilizumab (64).

As of May 1, 2021, 11 RCTs with tocilizumab (10 RCTs) and/or sarilumab (2 RCTs) were published in peer-reviewed journals (43, $58,65-73)$. Many of the observations made in the above reported Italian observational studies were confirmed in the RCTs. Studies were heterogenous in sample size, patient composition (e.g., age, sex, ethnicity), treatment protocol (e.g., dosage, number and timing of infusions), concomitant therapies (e.g., glucocorticoids, antivirals), and disease severity (e.g., respiratory status, oxygen requirement, inflammatory markers) (Table 2).

Many studies with negative results recruited fewer than 100 patients into the treatment $\operatorname{arm}(43,67,68,73)$, while the best outcomes were reported in the two largest trials (i.e., RECOVERY and REMAP-CAP) $(58,65)$, again suggesting that some inconclusive results may have suffered from low statistical power. Studies with worse results also included patients younger ( $<59$ years old) $(43,68-70)$ than other studies with better results (61-64 years old) $(58,65,66,72)$. Excluding RECOVERY, studies with overall negative outcomes included fewer men $(<70 \%)(43$, $67,69-71,73)$ compared to other studies with positive or partially positive outcomes $(\geq 70 \%)(65,66,72)$. Whereas successful trials included high numbers of Caucasian White patients $(\geq 70 \%)(58,65)$, worse results were obtained in trials with fewer White patients and relatively more Hispanic White and/or Black Afroamerican patients $(43,69-71)$, or with Asian patients $(67,68)$. Studies with poor outcomes also included numerous patients with comorbidities (e.g., obesity, hypertension, kidney impairment) $(43,68,69,71)$.

Treatment was given intravenously, at various doses (tocilizumab $400-800 \mathrm{mg}$ or $6-8 \mathrm{mg} / \mathrm{Kg}$, in $1-2$ infusions, at variable intervals ranging from 8 hours to 7 days; or sarilumab 200-400 mg in a single infusion), and was mostly started within the first 3 days of hospitalisation and/or within 2 weeks from symptom onset. Importantly, in severe-to-critical patients, treatment was successful if given within 24 hours from ICU admission (65), but not later (69).

In successful trials, standard of care included glucocorticoids, which were then administered to the great majority of the patients (> 80\%) $(58,65)$, whereas substantially fewer patients were cotreated with steroids in studies with negative results $(43,67,69,71$, 73). Antivirals, and in particular remdesivir, were given in variable proportion, without obvious implications in the overall outcome.

Most remarkably, best responses on disease progression and mortality were obtained in trials enrolling a substantial proportion of patients requiring $\mathrm{HFO}$ or NIV at baseline (> $40 \%$, ie. severe disease), a limited amount of patients requiring LFO $(<50 \%$, ie. moderate disease), and only a minority of patients requiring IMV ( $<30 \%$, ie. critical disease) $(58,65)$. Even in COVINTOC trial (68), an Indian RCT reporting overall negative or partial results, positive outcomes were observed when analysing specifically the "severe subgroup", in which these proportions were respected. As observed for earlier Italian studies (although the data here are largely lacking), a baseline median $\mathrm{PaO} 2 / \mathrm{FIO} 2$ (or $\mathrm{SaO} 2 / \mathrm{FIO} 2$ ) ratio $>110 \mathrm{mmHg}$ was reported in a successful trial (65), whereas values $>200 \mathrm{mmHg}$ were reported in negative trials $(69,73)$.

Concerning acute-phase reactants, in line with previous Italian cohort studies, trials with positive outcomes reported baseline median CRP values overall ranging between 120 and $155 \mathrm{mg} / \mathrm{L}$. In particular: good efficacy (i.e., significantly reduced progression and mortality) was reported in trials with CRP values between 130 and $150 \mathrm{mg} / \mathrm{L}$ (58, 65); partial efficacy (i.e., significantly reduced progression including ICU transfer and IMV rate, but not overall mortality) was observed in trials with CRP values between 120 and $125 \mathrm{mg} / \mathrm{L}(70,72)$ or around $155 \mathrm{mg} / \mathrm{L}$ (66); and no efficacy was obtained in trials with CRP values $\leq$ $115 \mathrm{mg} / \mathrm{L}(69,71,73)$ or $\geq 160 \mathrm{mg} / \mathrm{L}$ (43). Furthermore, whereas the two major successful trials largely included patients with only modestly increased levels of ferritin and D-dimer $(58,65)$, trials that reported poor outcomes mostly included patients with lower $(69,71$, 73 ) or higher (43) values of ferritin ( $<800$ or $>1200-1400 \mathrm{ng} / \mathrm{ml})$ and D-dimer $(<700$ or $>1000-1500 \mathrm{ng} / \mathrm{ml})$, and trials that reported partial efficacy showed dichotomous trends $(70,72)$.

No significant increase in serious adverse events associated with IL-6 inhibitors was noted. Indeed, in some trials, serious infections were numerically lower in the treatment arm $(66,71$, 72), and significantly lower in one study (71) (Table 2). Results from the MARIPOSA study suggest a more favourable profile for tocilizumab at a dose of $8 \mathrm{mg} / \mathrm{Kg}$ versus $4 \mathrm{mg} / \mathrm{Kg}$ in terms of lower mortality and fewer serious adverse events (74).

\section{CONFIRMATIONS FROM META- ANALYSES AND CURRENT GUIDELINES}

Early meta-analyses of the first published RCTs did not show significant differences in mortality with anti-IL-6R agents, although they showed significantly lower rates of ICU transfer, 
TABLE 2 | Randomised controlled trials with IL-6 inhibitors in COVID-19.

\begin{tabular}{|c|c|c|c|c|c|c|c|c|c|c|c|}
\hline $\begin{array}{l}\text { PROVED } \\
\text { EFFICACY }\end{array}$ & YES & YES & PARTIAL & NO & $\begin{array}{l}\text { NO (YES } \\
\text { in "severe } \\
\text { subgroup") }\end{array}$ & NO & NO & $\begin{array}{l}\text { NO } \\
\text { (PARTIAL) }\end{array}$ & NO & PARTIAL & NO \\
\hline \multicolumn{12}{|c|}{ TRIAL CHARACTERISTICS: } \\
\hline $\begin{array}{l}\text { Authors } \\
\text { [Ref.] }\end{array}$ & $\begin{array}{l}\text { Horby } \\
\text { et al. } \\
\text { (58) }\end{array}$ & $\begin{array}{l}\text { Gordon } \\
\text { et al. } \\
\text { (65) }\end{array}$ & $\begin{array}{l}\text { Rosas } \\
\text { et al. } \\
\text { (66) }\end{array}$ & $\begin{array}{l}\text { Wang } \\
\text { et al. } \\
\text { (67) }\end{array}$ & $\begin{array}{l}\text { Soin } \\
\text { et al. } \\
\text { (68) }\end{array}$ & $\begin{array}{l}\text { Lescure } \\
\text { et al. } \\
\text { (69) }\end{array}$ & $\begin{array}{l}\text { Veiga } \\
\text { et al. } \\
\text { (43) }\end{array}$ & $\begin{array}{l}\text { Salama } \\
\text { et al. } \\
\text { (70) }\end{array}$ & $\begin{array}{l}\text { Stone } \\
\text { et al. } \\
\text { (71) }\end{array}$ & $\begin{array}{l}\text { Hermine } \\
\text { et al. } \\
\text { (72) }\end{array}$ & $\begin{array}{l}\text { Salvarani } \\
\text { et al. } \\
\text { (73) }\end{array}$ \\
\hline $\begin{array}{l}\text { Trial name } \\
\text { (Geographic } \\
\text { area) }\end{array}$ & $\begin{array}{l}\text { RECOVERY } \\
\text { (UK) }\end{array}$ & $\begin{array}{l}\text { REMAP-CAP } \\
\text { (Multinational) }\end{array}$ & $\begin{array}{l}\text { COVACTA } \\
\text { (Multinational) }\end{array}$ & (China) & $\begin{array}{l}\text { COVINTOC } \\
\text { (India) }\end{array}$ & (Multinational) & $\begin{array}{l}\text { TOCIBRAS } \\
\text { (Brazil) }\end{array}$ & $\begin{array}{l}\text { EMPACTA } \\
\text { (Multinational) }\end{array}$ & $\begin{array}{l}\text { BACC Bay } \\
\text { (USA) }\end{array}$ & $\begin{array}{l}\text { CORIMUNO- } \\
\text { TOCl } 1 \\
\text { (France) }\end{array}$ & $\begin{array}{l}\text { RCT-TCZ- } \\
\text { COVID-19 } \\
\text { (Italy) }\end{array}$ \\
\hline $\begin{array}{l}\text { Date of (online) } \\
\text { publication } \\
\text { (preprints or } \\
\text { updates) }\end{array}$ & $\begin{array}{l}\text { May 1, } \\
2021 \\
\text { (preprint } \\
\text { Feb 11, } \\
2021 \text { ) }\end{array}$ & $\begin{array}{l}\text { Apr 22, } \\
2021 \\
\text { (preprint } \\
\text { Jan 9, } \\
\text { 2021) }\end{array}$ & $\begin{array}{l}\text { Apr 22, } \\
2021 \\
\text { (preprint } \\
\text { Sep 12, } \\
\text { 2020) }\end{array}$ & $\begin{array}{l}\text { Mar 9, } \\
2021\end{array}$ & $\begin{array}{l}\text { March 4, } \\
2021\end{array}$ & $\begin{array}{l}\text { March 4, } \\
2021 \\
\text { (preprint } \\
\text { Feb 3, } \\
\text { 2021) }\end{array}$ & $\begin{array}{l}\text { Jan 20, } \\
2021\end{array}$ & $\begin{array}{l}\text { Dec 17, } \\
2020\end{array}$ & $\begin{array}{l}\text { Oct 21, } \\
2020 \\
\text { (updated } \\
\text { Nov 19, } \\
\text { 2020) }\end{array}$ & $\begin{array}{l}\text { Oct 20, } \\
2020 \\
\text { (corrected } \\
\text { Jan 4, } \\
2021 \text { ) }\end{array}$ & $\begin{array}{l}\text { Oct } 20 \text {, } \\
2020\end{array}$ \\
\hline $\begin{array}{l}\text { Period of } \\
\text { recruitment }\end{array}$ & $\begin{array}{l}\text { Apr 23, } 2020 \text { - } \\
\text { Jan 24, } 2021\end{array}$ & $\begin{array}{l}\text { Mar } 9- \\
\text { Nov } 19,2020\end{array}$ & $\begin{array}{l}\text { Apr } 3- \\
\text { May 28, } 2020\end{array}$ & $?$ & $\begin{array}{l}\text { May } 30- \\
\text { Aug 31, } 2020\end{array}$ & $\begin{array}{l}\text { Mar 28 - } \\
\text { Jul 3, } 2020\end{array}$ & $\begin{array}{l}\text { May } 8 \text { - } \\
\text { Jul 17, } 2020\end{array}$ & $\begin{array}{l}?- \\
\text { Sep 30, } 2020\end{array}$ & $\begin{array}{l}\text { Apr } 20- \\
\text { Jun 15, } 2020\end{array}$ & $\begin{array}{l}\text { Mar 31- } \\
\text { Apr 18, } 2020\end{array}$ & $\begin{array}{l}\text { Mar 31- } \\
\text { Jun 11, } 2020\end{array}$ \\
\hline $\begin{array}{l}\text { Design of the } \\
\text { study }\end{array}$ & Open-label & Open-label & Double-blind & Open-label & Open-label & Double-blind & Open-label & Double-blind & Double-blind & Open-label & Open-label \\
\hline $\begin{array}{l}\text { Modified } \\
\text { intention-to-treat } \\
\text { population }\end{array}$ & 4116 & 803 & 438 & 55 & 180 & 416 & 129 & 377 & 242 & 131 & 123 \\
\hline $\begin{array}{l}\text { N. Patients in } \\
\text { the } \\
\text { treatment arm }\end{array}$ & 2022 & $\begin{array}{l}401 \\
{[353 \text { (toci) plus }} \\
48 \text { (sari)] }\end{array}$ & 294 & 33 & 91 & $\begin{array}{l}159(200 \mathrm{mg} \\
\text { arm) } \\
\text { and } \\
174(400 \mathrm{mg} \\
\text { arm) }\end{array}$ & 65 & 249 & 161 & 64 & 60 \\
\hline $\begin{array}{l}\text { Treatment arm } \\
\text { (route, dosage, } \\
\text { number of } \\
\text { doses, intervals) }\end{array}$ & $\begin{array}{l}\text { Tocilizumab } \\
\text { IV } 400-800 \mathrm{mg} \\
(\approx 8 \mathrm{mg} / \mathrm{Kg}) \\
(1+1 \text { optional } \\
\text { after } 12-24 \mathrm{~h})\end{array}$ & $\begin{array}{l}\text { Tocilizumab } \\
\text { IV } 8 \text { mg/kg } \\
\text { (max } 800 \text { mg) } \\
\text { (1+ } 1 \text { optional } \\
\text { after } 12-24 h) ; \\
\text { or Sarilumab } \\
\text { IV } 400 \text { mg } \\
\text { (single dose) }\end{array}$ & $\begin{array}{l}\text { Tocilizumab } \\
\text { IV } 6 \text { mg/Kg } \\
\text { (max } 480 \text { mg) } \\
\text { ( } 1+1 \text { optional } \\
\text { after } 8-24 \mathrm{~h} \text { ) }\end{array}$ & $\begin{array}{l}\text { Tocilizumab } \\
\text { IV } 400 \text { mg } \\
\text { (1+1 optional } \\
\text { after } 24 \mathrm{~h})\end{array}$ & $\begin{array}{l}\text { Tocilizumab } \\
\text { IV } 6 \mathrm{mg} / \mathrm{Kg} \\
\text { (max } 480 \mathrm{mg}) \\
(1+1 \\
\text { optional } \\
\text { after 12- } \\
\text { 168h) }\end{array}$ & $\begin{array}{l}\text { Sarilumab } \\
\text { IV 200-400 mg } \\
\text { (single dose) }\end{array}$ & $\begin{array}{l}\text { Tocilizumab } \\
\text { IV } 8 \mathrm{mg} / \mathrm{Kg} \\
\text { (single dose) }\end{array}$ & $\begin{array}{l}\text { Tocilizumab } \\
\text { IV } 8 \mathrm{mg} / \mathrm{Kg} \\
\text { (1+1 optional } \\
\text { after } 8-24 \mathrm{~h})\end{array}$ & $\begin{array}{l}\text { Tocilizumab } \\
\text { IV } 8 \mathrm{mg} / \mathrm{Kg} \\
\text { (max } 800 \\
\mathrm{mg}) \\
\text { (single dose) }\end{array}$ & $\begin{array}{l}\text { Tocilizumab } \\
\text { IV } 8 \mathrm{mg} / \mathrm{Kg} \\
\text { ( } 1+1 \text { optional } \\
\text { after } 48 \mathrm{~h})\end{array}$ & $\begin{array}{l}\text { Tocilizumab } \\
\text { IV } 8 \mathrm{mg} / \mathrm{Kg} \\
\text { (max } 800 \\
\mathrm{mg} \text { ) } \\
+ \text { second } \\
\text { dose after } \\
12 \mathrm{~h}\end{array}$ \\
\hline \multicolumn{12}{|c|}{ PATIENT CHARACTERISTICS (treatment arm): } \\
\hline $\begin{array}{l}\text { (Median) Age } \\
\text { (years old) }\end{array}$ & 63 & (mean) 61.5-63 & (mean) 61 & 63.5 & 56 & $58-58$ & (mean) 57 & (mean) 56 & 62 & 64 & 61.5 \\
\hline Male sex (\%) & 66 & $74-81$ & 70 & 53 & 84 & $68-57$ & (mean) 68 & 60 & 60 & 70 & 67 \\
\hline $\begin{array}{l}\text { Race/Ethnicity } \\
\text { (\%) }\end{array}$ & $\begin{array}{l}\text { White } 76, \text { Black } \\
\text { +Asian+Other } 18, \\
\text { Unknown } 7\end{array}$ & $\begin{array}{l}\text { White 70-74, Asian } \\
\text { 18-21, Black 5-3, } \\
\text { Other 7-3 }\end{array}$ & $\begin{array}{l}\text { White 60, } \\
\text { Black 14, } \\
\text { Asian } 9.5 \text {, } \\
\text { Other } 3.5 \text {, } \\
\text { Unknown } 13\end{array}$ & Asian 100 & Asian 100 & $\begin{array}{l}\text { White 79-74 } \\
\text { (Hispanic 33- } \\
\text { 38), Black 2-3, } \\
\text { Asian 3-5, } \\
\text { Other 16-18 }\end{array}$ & $\begin{array}{l}\text { Hispanic } \\
100\end{array}$ & $\begin{array}{l}\text { White 68.5 } \\
\text { (Hispanic } \\
\mathbf{5 7 . 5 ) , ~ B l a c k ~} \\
\text { 14, } \\
\text { Other 13.5, } \\
\text { Unknown } 4\end{array}$ & $\begin{array}{l}\text { White 44, } \\
\text { Black 15, } \\
\text { Asian 4, } \\
\text { Other 23, } \\
\text { Unknown } 14\end{array}$ & & \\
\hline $\begin{array}{l}\text { (Median) BMI } \\
\text { Obesity (\%) }\end{array}$ & & $30.5-29$ & & & (mean) 27 & & & (mean) 32 & $\begin{array}{l}30 \\
50\end{array}$ & 28 & \\
\hline $\begin{array}{l}\text { Obesity (\%) } \\
\text { Type-2 } \\
\text { Diabetes (\%) }\end{array}$ & 28 & & 36 & 12 & 34 & $\begin{array}{l}23-21 \\
28-27\end{array}$ & $\begin{array}{l}23 \\
34\end{array}$ & & $\begin{array}{l}50 \\
28\end{array}$ & 33 & $\begin{array}{l}28 \\
17\end{array}$ \\
\hline
\end{tabular}


TABLE 2 | Continued

\begin{tabular}{|c|c|c|c|c|c|c|c|c|c|c|c|}
\hline $\begin{array}{l}\text { PROVED } \\
\text { EFFICACY }\end{array}$ & YES & YES & PARTIAL & NO & $\begin{array}{l}\text { NO (YES } \\
\text { in "severe } \\
\text { subgroup") }\end{array}$ & NO & NO & $\begin{array}{l}\text { NO } \\
\text { (PARTIAL) }\end{array}$ & NO & PARTIAL & NO \\
\hline $\begin{array}{l}\text { Hypertension } \\
\text { (\%) }\end{array}$ & & & 60.5 & 29 & 40 & $43-40$ & 46 & & 50 & & 45 \\
\hline $\begin{array}{l}\text { Cardiovascular } \\
\text { impairment (\%) }\end{array}$ & 22 & & 30 & & 16 & $4-5$ & 12 & & 20 & 33 & \\
\hline $\begin{array}{l}\text { Chronic Lung } \\
\text { Disease (\%) }\end{array}$ & 23 & & 17 & & 5 & $9-7$ & 9 & & 18 & 13 & 3 \\
\hline $\begin{array}{l}\text { (Severe) Hepatic } \\
\text { impairment (\%) }\end{array}$ & 1 & & 2 & & & & & & & & \\
\hline $\begin{array}{l}\text { (Severe) Kidney } \\
\text { impairment (\%) }\end{array}$ & 1 & & & & 4 & $4-3$ & 8 & & 18 & 8 & \\
\hline $\begin{array}{l}\text { N. Days from } \\
\text { hospitalization }\end{array}$ & 2 & $\begin{array}{l}1-1 \\
\text { (1 day - median } 14 \\
\text { hours - from ICU } \\
\text { admission) }\end{array}$ & 3 & & & $\begin{array}{l}3-2 \\
(2 \text { days from } \\
\text { ICU } \\
\text { admission) }\end{array}$ & & & & 1 & 2 \\
\hline $\begin{array}{l}\text { N. Days from } \\
\text { symptom onset }\end{array}$ & 9 & & 12 & 20 & & $5-4$ & 10 & & 9 & 10 & 7 \\
\hline $\begin{array}{l}\text { Glucocorticoids } \\
\text { (\%) }\end{array}$ & 82 & $87-91$ & 34 & 15 & $\begin{array}{l}91 \text { (96 in } \\
\text { "severe") }\end{array}$ & $\begin{array}{l}16-24 \\
\text { (varying during } \\
\text { the study) }\end{array}$ & 69 & 80 & 11 & 33 & 0 \\
\hline Remdesivir (\%) & & 33 & & & $\begin{array}{l}43 \text { ( } 42 \text { in } \\
\text { "severe") }\end{array}$ & $<1$ & 0 & 53 & 33 & & 0 \\
\hline $\begin{array}{l}\text { (Median) } \mathrm{PaO} 2 / \\
\mathrm{FlO} 2 \text { ratio } \\
\text { (mmHg) }\end{array}$ & & $115-126$ & & & & $\begin{array}{l}\text { (SpO2/FiO2 } \\
\text { ratio) 230- } \\
237.5\end{array}$ & & & & & 262.5 \\
\hline Ambient air (\%) & 0.5 & $<1-0$ & 3 & 23 & $\begin{array}{l}11 \text { (2 in } \\
\text { "severe") }\end{array}$ & $1-0$ & 0 & 9 & 14 & 0 & 0 \\
\hline LFO (\%) & 45.5 & $<1-0$ & 27 & 68 & $\begin{array}{l}53 \text { (42 in } \\
\text { "severe") }\end{array}$ & $70-74$ & 60 & 65 & 83 & 100 & 100 \\
\hline HFO or NIV (\%) & 41 & $71-83$ & 32 & 9 & $\begin{array}{l}31 \text { (46 in } \\
\text { "severe") }\end{array}$ & $18-12$ & 23 & 26 & 3 & 0 & 0 \\
\hline $\begin{array}{l}\text { IMV or ECMO } \\
\text { (\%) }\end{array}$ & 13 & $29-17$ & 38 & 0 & $\begin{array}{l}5 \text { (10 in } \\
\text { "severe") }\end{array}$ & $11-14$ & 17 & 0 & 0 & 0 & 0 \\
\hline $\begin{array}{l}\text { (Median) CRP } \\
\text { (mg/l) }\end{array}$ & 143 & $150-136$ & 157 & & $\begin{array}{l}\text { (mean) } 111 \\
\text { (N/A in } \\
\text { "severe") }\end{array}$ & $94-96$ & (mean) 160 & 124.5 & 116 & 119.5 & 105 \\
\hline $\begin{array}{l}\text { (Median) Ferritin } \\
(\mathrm{ng} / \mathrm{ml})\end{array}$ & 947 & & (mean) 3066.5 & & $\begin{array}{l}\text { (mean) } 921 \\
\text { (N/A in } \\
\text { "severe") }\end{array}$ & $695-737$ & (mean) 1271 & 624 & 723 & 1292 & 646 \\
\hline $\begin{array}{l}\text { (Median) D- } \\
\text { dimer (ng/ml) }\end{array}$ & & $832-828$ & & & & $480-540$ & 1000 & 1600 & 857 & 869 & 756 \\
\hline \multicolumn{12}{|c|}{ OUTCOMES (treatment vs. standard-of-care): } \\
\hline $\begin{array}{l}\text { Clinical status or } \\
\text { functional } \\
\text { Improvement } \\
\text { (\%) }\end{array}$ & & $\begin{array}{l}\text { 21-day median } \\
\text { organ support- } \\
\text { free days: } \\
10-11 \text { vs. } 0 \\
\text { (Adjusted OR }\end{array}$ & $\begin{array}{l}\text { 28-day median } \\
\text { ordinal scale: } \\
1 \text { vs. } 2 \text { ( } p=N S \text { ); } \\
\text { Median time to } \\
\text { improvement: }\end{array}$ & $\begin{array}{l}\text { 14-day, "cure } \\
\text { rate": } 94 \text { vs. } \\
87 \text { ( } p=N S) ; \\
\text { 14-day } \\
\text { hypoxia }\end{array}$ & & $\begin{array}{l}\text { Median time } \\
\text { to } \\
\geq 2 \text {-point } \\
\text { improvement: } \\
10-10 \text { vs. } 12\end{array}$ & & $\begin{array}{l}\text { Median time } \\
\text { to } \\
\text { improvement: } \\
6 \text { vs. } 7 \text { days } \\
\text { (HR 1.15) }\end{array}$ & $\begin{array}{l}91 \text { vs. } 89 \\
\text { (HR 1.08); } \\
\text { Weaned } \\
\text { from }\end{array}$ & $\begin{array}{l}\text { Weaned from } \\
\text { oxygen: } \\
89 \text { vs. } 75\end{array}$ & \\
\hline
\end{tabular}


TABLE 2 | Continued

PROVED YES

YES

PARTIAL

No

NO (YES

No

No

NO

(PARTIAL)

No

PARTIAL

NO

subgroup")

\begin{tabular}{ll}
\hline & \\
(28-day) & 57 vs. 50 \\
Discharge (\%) & $(\mathrm{p}<0.0001)$ (RR \\
& $1.22) ;$ \\
& Median time to \\
& discharge: 19 \\
& vs. $>28$ days) \\
(28-day) &
\end{tabular}

$\begin{array}{lll}\begin{array}{ll}\text { 1.64-1.76, } \\ \text { probability of } \\ \text { superiority >99\%) }\end{array} & \begin{array}{l}14 \text { vs. } 18 \text { days (HR } \\ 1.26)\end{array} & \begin{array}{l}\text { recovery rate: } \\ 92 \text { vs. } 60, \\ \mathrm{p}=0.033)\end{array} \\ & \begin{array}{l}\text { Median time to } \\ \text { discharge: }\end{array} & \begin{array}{l}\text { Time to } \\ \text { discharge: } 26\end{array} \\ & 20 \text { vs. } 28 \text { days (HR } & \text { vs. } 24 \text { days } \\ & \begin{array}{l}1.35, \text { nominal } \\ \mathrm{p}=0.04 \text { in preprint } \\ \text { version) }\end{array} & \\ & \end{array}$

Progression (\%)

ICU transfer:

21 vs. 36

\section{2 vs. 18 \\ (p=NS); \\ 16 vs. 34 \\ $(p=0.044)$ in}

"severe"

days
(HR 1.03-1.14,
$p=N S)$

$\mathrm{p}=\mathrm{NS}$ )

15 vs. 15

( $p=N S$ )

(RR 0.79,

(28-day) IMV + 35 vs. 42

death (\%) (RR 0.84 ,

p<0.0001)

(28-day)

31 vs. 35

Mortality $(\%)$

(HR 0.85 ,

$\mathrm{p}=0.0028$ );

(steroids vs. no

steroids: death

RR

0.79 vs. 1.16 ,

interaction

$\mathrm{p}=0.01$

Serious adverse 3 vs. 0

events (\%)

Serious 3 vs. 0

9-0 vs. 11

35 vs. 38.5

1-0 vs. 0

21 vs. 26

ICU+IMV+death

(clinical failure): 29

vs. 42

(HR 0.61, nominal

$\mathrm{p}=0.03$ in preprint

(21-day)

28-22 vs. $36 \quad$ (p=NS)

(adjusted OR 1.64-

, probability of

superiority

$>99 \%)$

LEGEND OF COLOURS: Green: numbers falling within the thresholds herein suggested (optimal trial and patient characteristics), in relation with statistically significant results, and significant benefit on overall mortality (positive outcomes). Blue: numbers falling borderline (suboptimal trial and patient characteristics), in relation with numerically (i.e., >20\% differences) but not statistically significant results, and significant benefit on composite endpoints (IMV+death) but not on overall mortality (partially positive outcomes). Red: numbers falling outside of the thresholds (non-optimal trial and patient characteristics), in relation with non-significant results, and no benefit on either composite endpoints or overall mortality

(negative outcomes).

$H R$, hazard ratio; $O R$, odds ratio; $R R$, relative risk; NS, not significant.

$\begin{array}{lll}19 \text { vs. } 17 & \text { (14-day) } & \text { (30-day) } \\ \text { (HR 1.11, } & \text { ventilator-free } & \text { ICU } \\ \mathrm{p}=\text { NS) } & \text { survival: } & \text { transfer: } \\ & 24 \text { vs. } 36 & 10 \text { vs. } 8 \\ & \text { (HR } 0.58, & \text { (RR 1.26) } \\ & \text { probability of } & \\ & \text { HR<1 }=95 \%) & \end{array}$

\begin{tabular}{|c|c|c|c|c|c|c|}
\hline & & $\begin{array}{l}\text { (15-day) } \\
28 \text { vs. } 20 \\
\text { (OR 1.54, } \\
p=N S \text { ) }\end{array}$ & $\begin{array}{l}12 \text { vs. } 19 \\
\text { (HR 0.56, } \\
\mathrm{p}=0.04)\end{array}$ & $\begin{array}{l}11 \text { vs. } 12.5 \\
\text { (HR } 0.83, \\
\mathrm{p}=\mathrm{NS} \text { ) }\end{array}$ & $\begin{array}{l}\text { (14-day) } \\
17 \text { vs. } 27 \\
\text { (HR } 0.58, \\
\text { probability of } \\
\text { HR<1 = } \\
92.5 \%)\end{array}$ & \\
\hline $\begin{array}{l}12 \text { vs. } 17 \\
(p=N S) ; \\
16 \text { vs } 34 \text { in } \\
\text { "severe" }\end{array}$ & $\begin{array}{l}\text { (29-day) } \\
10-8 \text { vs. } 8 \\
(p=N S)\end{array}$ & $\begin{array}{l}21 \text { vs. } 9 \\
\text { (OR 2.70, } \\
p=0.07 \text { ) }\end{array}$ & $\begin{array}{l}10 \text { vs. } 9 \\
(p=N S)\end{array}$ & $\begin{array}{l}6 \text { vs. } 4 \\
\text { (HR 1.52) }\end{array}$ & $\begin{array}{l}11 \text { vs. } 12 \\
\text { (HR 0.92, } \\
p=N S)\end{array}$ & $\begin{array}{l}\text { (30-day) } \\
3 \text { vs. 1.5 } \\
\text { (RR 2.10, } \\
\text { p=NS) }\end{array}$ \\
\hline
\end{tabular}

$\begin{array}{llll}\text { Median time } & 91 \text { vs. } 89 & 83 \text { vs. } 73 & \text { (30-day) } \\ \text { to discharge: } & \text { (HR 1.08) } & & 90 \text { vs. } 92 \\ 6 \text { vs. } 7.5 \text { days } & & & \text { (RR 0.98) } \\ \text { (HR 1.16) } & & & \end{array}$

6 vs. 7.5 days

0.98)

$(p=0.044)$

$$
\begin{aligned}
& 0 \text { vs. } 3 \\
& 20 \text { vs. } 17 \\
& \text { 26-29 vs. } 24 \quad 16 \text { vs. } 11 \\
& (p=N S) \\
& 15 \text { vs. } 20 \\
& 5 \text { vs. } 7
\end{aligned}
$$


IMV, and composite outcome of IMV or death, along with a lower rate of serious infections $(22,75,76)$. This could have been due to the small size and composite primary endpoints of early trials, probably underpowered to detect differences in mortality, and to the high heterogeneity among the studies. However, subsequent metaanalyses of more recently completed RCTs including RECOVERY, or even unpublished data through searches of electronic databases and contact with experts, ultimately concluded that, in patients hospitalised with COVID-19, treatment with IL-6 antagonists, and in particular tocilizumab (with much higher certainty than sarilumab, but not siltuximab), results into a significant reduction in 28-day all-cause mortality $(2,32,58)$, in line with previous metaanalyses of observational cohort studies $(22,77,78)$. Specifically, high probability of reduced risk of mortality and clinically meaningful mortality benefit was observed in patients receiving concomitant glucocorticoids and non-invasive ventilation or highflow oxygen, with no need of IMV or cardiovascular support $(32,79)$. A significant interaction was seen in the subgroup analysis in regard to concomitant steroid therapy (2), while other meta-analyses confirmed the independent strong benefit of steroid therapy, particularly low-dose dexametasone (not high-dose methylprednisolone or hydrocortisone), on survival of severe and critical patients (80). Lower odds of mortality with tocilizumab were observed in patients with CRP values between 75 and $150 \mathrm{mg} / \mathrm{L}$ compared to patients with lower or higher levels; yet, a statistical significance was not reached (32).

According to the NIH COVID-19 treatment guidelines (81), largely based on the findings and recruiting criteria of RECOVERY and REMAP-CAP trials, tocilizumab (at a single intravenous dose of $8 \mathrm{mg} / \mathrm{Kg}$ actual body weight up to $800 \mathrm{mg}$ ) or sarilumab (in case tocilizumab is not available, at a single intravenous dose of $400 \mathrm{mg}$ ) are recommended, in combination with dexamethasone $(6 \mathrm{mg}$ per day, intravenously, for up to 10 days), for recently hospitalised patients (within 3 days from hospital admission and/or within 24 hours from ICU admission), with a rapid respiratory decompensation requiring increasing amounts of oxygen (in particular, HFO or NIV, or soon after starting IMV), and substantially increased markers of inflammation (e.g., CRP $\geq 75 \mathrm{mg} / \mathrm{L}$ ).

\section{THERAPEUTIC WINDOWS: TO CURE THE RIGHT PATIENT AT THE RIGHT TIME WE NEED CLEAR CUT-OFFS}

A correct stratification of the patients is crucial for achieving satisfactory results with tocilizumab in the treatment of COVID19, in clinical trials as in clinical practise. Although only large individual patient data meta-analyses would best indicate which patients are more likely to benefit from IL-6R blockade, from this review of the data we aimed to identify, for some important respiratory and inflammatory parameters, the minimum and maximum thresholds between which to build up a therapeutic window.

Relatively simple numerical cut-offs would actually reflect the complexity and heterogeneity of disease biology, helping to recognize the stage and progression of the disease, the prevailing pathogenic aspects, the predominant inflammatory patterns, and the actual significance of IL- 6 in a given patient.

IL-6 levels are significantly correlated with worsening oxygen exchange in the lungs of COVID-19 patients and are major predictors of disease progression and mortality (23). Plasma levels of IL- $6<7-10 \mathrm{pg} / \mathrm{ml}$ are found in healthy subjects (and presumably asymptomatic carriers); $<20 \mathrm{pg} / \mathrm{ml}$ (median $17 \mathrm{pg} / \mathrm{ml}$ ) in low-risk patients (i.e., mild-moderate disease); > $35 \mathrm{pg} / \mathrm{ml}$ (around 2/3-fold higher median values) in patients at risk of progression (i.e., moderate-to-severe disease); > $55 \mathrm{pg} / \mathrm{ml}$ in patients with a complicated course (i.e., severe disease); and $>80 \mathrm{pg} / \mathrm{ml}$ (median values $86-91 \mathrm{pg} / \mathrm{ml}$ ) in patients developing severe respiratory failure requiring ventilatory support and at high risk of in-hospital death (i.e., severe-to-critical and critical disease) (82-87). These thresholds are in line with results of univariate and multivariate analysis of the Italian observational studies exposed above $(51,59)$. Interestingly, daily monitoring of IL- 6 levels showed that, in severe progressive forms, IL-6 increases up to $85-90 \mathrm{pg} / \mathrm{ml}$ at $6-7$ days of hospitalisation, and reaching these values precedes a further increase to peak concentrations above $120-300 \mathrm{pg} / \mathrm{ml}$ on days 910 (88). Acting within 6 days of hospitalisation in severe patients, identified by means of baseline IL- 6 values between 35 and 90 pg/ $\mathrm{ml}$, may therefore represent the right time to interrupt the inflammatory cascade and prevent lethal cytokine storm.

A good response to IL-6R blockade is expected when IL-6 elevation is accompanied at baseline by an increase in CRP values. Higher levels of both IL-6 and CRP are, in fact, associated with disease severity in COVID-19 (89). Indeed, cytokine blockade was not effective in COVID-19-unrelated ARDS (showing higher IL-6 levels but much lower CRP values as compared to severe COVID19), while it is effective in CAR T-cell induced CRS (showing high concentrations of both IL-6 and CRP) (90). On the other hand, tocilizumab does not reduce serum IL-6 concentrations, which are instead variably increased upon treatment, but it does efficiently reduce CRP levels, which reflect IL-6 bioactivity. CRP levels $<80$ $100 \mathrm{mg} / \mathrm{L}$ (median $77 \mathrm{mg} / \mathrm{L}$ ) are found in patients at low risk of progression (i.e., mild-moderate disease), whereas CRP levels > 180-200 mg/L (median $194 \mathrm{mg} / \mathrm{L}$ ) are detected in patients with high rates of mortality (i.e., critical disease) $(86,87)$. From the observational studies and RCTs reported above, it emerges that patients with CRP values between 120 and $160 \mathrm{mg} / \mathrm{L}$ are actually those with a severe progressive disease who are most likely to respond successfully to IL-6 inhibitors.

In regard to serum ferritin levels, observations from the aforementioned retrospective cohort studies and RCTs seem to confirm other reports $(91,92)$, according to which low-risk patients can be identified for values $<400-800 \mathrm{ng} / \mathrm{ml}$ (i.e., mild-moderate disease); patients at high risk of progression for values between 800 and $1400-1600 \mathrm{ng} / \mathrm{ml}$ (i.e., severe progressive disease); and patients at high risk of death for values $>1600-2000 \mathrm{ng} / \mathrm{ml}$ (i.e., critical disease). Severe hyperferritinemia ( $>1600-2000 \mathrm{ng} / \mathrm{ml}$ ) is a criterion for MAS $(93,94)$, and probably indicates an overt state of MAS-like hyperinflammation in COVID-19 that specifically occurs in critically-ill patients (19). Due to the postulated regulatory role of IL-6 at this stage, tocilizumab would be no longer effective in these cases; conversely, activation of the NLR family pyrin domain 
containing 3 (NLRP3) inflammasome, which is typical of hyperferritinemic syndromes, would be crucial also in critical COVID-19 (7), and therapies targeting IL-1, such as anakinra, could still be effective (95). In addition to being associated with intense macrophage activation and release of IL-1, high ferritin levels are also associated with severe hepatocellular damage and LDH elevation, as well as with development of diffuse microthrombosis and coagulopathy with severely increased Ddimer concentrations (19). In fact, high D-dimer levels were found to predict mortality and poor response to tocilizumab in COVID-19 patients $(61,96,97)$; indeed, treatment with tocilizumab could even lead to a further increase in D-dimer, despite the decrease in fibrinogen $(52,63)$. Altogether, COVID-19 patients characterised by serum ferritin levels > 1600-2000 ng/mL, LDH > 500-550 U/L, and D-dimer > 3000-5000 ng/mL, are most likely hyperinflamed and critically-ill patients who do not respond to IL6R blockade (but may still respond to IL-1 blockade). It remains to be assessed whether, in addition to these absolute thresholds, the ratio of CRP to ferritin (or D-dimer) may inform the advisability of using tocilizumab or anakinra (or full-dose anticoagulants). Most recently, the soluble urokinase plasminogen activator receptor (suPAR) has been proposed as an alternative and more sensitive biomarker for early identification of those COVID-19 patients at risk of progression who release high amounts of alarmins S100A8/ A9 and IL- $1 \alpha$ and particularly respond to anakinra, thus allowing the extension of the use of anakinra even to more moderate forms of disease (98).

Concerning respiratory parameters and oxygen requirements, as exposed in the previous paragraphs, RCTs and meta-analyses have concluded that the best responders to IL-6 inhibitors, particularly tocilizumab, are hypoxic and severely progressing patients requiring high-flow oxygen or non-invasive ventilation, while from retrospective cohort studies it can be suggested that this subgroup basically corresponds to patients with values of $\mathrm{PaO} 2 / \mathrm{FIO} 2$ ratio between 100 and $200 \mathrm{mmHg}$. In this subset of patients, median values of CRP, ferritin, D-dimer, LDH and neutrophil-to-lymphocyte ratio in blood actually fall within the optimal ranges proposed here $(47,59,95)$. By contrast, criticallyill patients (showing values of $\mathrm{PaO} 2 / \mathrm{FIO} 2$ ratio $<100 \mathrm{mmHg}$ and requiring IMV) and mild-moderate patients (showing values of $\mathrm{PaO} 2 / \mathrm{FIO} 2$ ratio $>200-300 \mathrm{mmHg}$ and requiring low-flow or no oxygen) generally show poor responses to IL-6 inhibitors. Nevertheless, results from a large RCT evaluating sarilumab recently posted as a preprint (99) seem to suggest that IL-6 inhibitors can reduce the risk of death by up to half even in ICU patients undergoing IMV, in case these are co-treated with steroids and still show relatively low median values of IL-6 and $\mathrm{CRP}$, substantially falling within our proposed response ranges.

A schematic summary of the suggested items and cut-offs to consider in COVID-19 to discern good and bad responders to tocilizumab (and other agents) is provided in Figure 3.

\section{GOING BEYOND IL-6}

In managing COVID-19, growing interest is turning to Janus kinase (JAK) inhibitors. Among these, baricitinib was originally identified using artificial intelligence as a suitable candidate for COVID-19 treatment, due to its ability to bind with high affinity to some key regulators of clathrin-mediated endocytosis, namely AP2-associated protein kinase-1 (AAK1) and possibly cyclic Gassociated kinase (GAK), thus inhibiting the entry of SARSCoV-2 into cells and the intracellular assembly of virus particles (100). Such antiviral effects were more recently demonstrated in vitro using $3 \mathrm{D}$ cultures of primary human liver cells, in which baricitinib was able to reduce SARS-CoV-2 infectivity and viral load by directly inhibiting numb-associated kinases and impeding IFN 22 -mediated induction of the SARS-CoV-2 receptor angiotensin-converting enzyme 2 (ACE2) (101). Plasma concentrations of baricitinib at therapeutic doses would be sufficient to exert antiviral effects in vivo (100).

Moreover, and perhaps most importantly, JAK inhibitors exert multidirectional anti-inflammatory effects by simultaneously inhibiting intracellular signals induced by several cytokines. Acting on JAK1 would broadly interfere with the signalling downstream of cytokine receptors belonging to the gp130 family (e.g., IL-6, IL-27), IFN family (e.g., IFN $\gamma$ ), $\gamma$-chain family (e.g., IL4, IL-9, IL-7) and others (e.g., thymic stromal lymphopoietin, IL13 , G-CSF); acting on JAK2 would specifically interfere with GMCSF, IL-23, IL-12 and IL-6 signals; while acting on JAK3 would more potently interfere with the $\gamma$-chain receptor family (102).

Different JAK inhibitors have been proposed for the treatment of COVID-19, namely: JAK1/JAK2/TYK2 inhibitors (e.g., baricitinib, ruxolitinib) (103-109), selective JAK2 inhibitors (e.g., fedratinib) (110), and - more recently - JAK1/JAK3 inhibitors (e.g., tofacitinib) (111). Probably due to their ability to block various inflammatory pathways including not only IL-6/ STAT3 and TH17 (advanced stages), but also TH9 and TH1/THGM pathways (upstream stages) (Figure 2) (7, 104-106), in addition to their presumed direct effects on viral infection and replication (early stages) $(100,101)$, JAK inhibitors appeared from both observational studies $(103,104,106)$ and RCTs $(107-$ $109,111)$ to be effective not only in severe forms of COVID-19, but also in patients with moderate disease (Figure 3), the latters representing the vast majority of patients in these studies (Table 3). Furthermore, baricitinib may work even in the absence of concomitant steroids, and in series involving more women and younger patients $(103,104,108,109)$. Nevertheless, as with tocilizumab, the most significant results with baricitinib (on time-to-recovery or all-cause mortality, according to ACTT2 and COV-BARRIER trials, respectively) were obtained in patients with severe disease requiring at baseline HFO or NIV (81, 108, 109) (Table 3).

IL-1 inhibitors, in particular the recombinant IL- $1 \alpha / \beta$ receptor antagonist anakinra, have been shown in observational studies to reduce mortality in both severe and critical disease $(112,113)$ (Figure 3). Whereas both IL-6 and IL-1 inhibitors would have a significant impact on mortality in patients showing a $\mathrm{PaO} 2 / \mathrm{FIO} 2$ ratio $\geq 100 \mathrm{mmHg}$, only IL-1 inhibitors would remain effective in patients with a $\mathrm{PaO} 2 / \mathrm{FIO} 2$ ratio $<100 \mathrm{mmHg}$ (95). In fact, as previously mentioned, critical COVID-19 assumes the connotations of a hyperferritinemic syndrome or an autoinflammatory febrile disease, in which an aberrant activation of the NLRP3 inflammasome, in this case resulting 


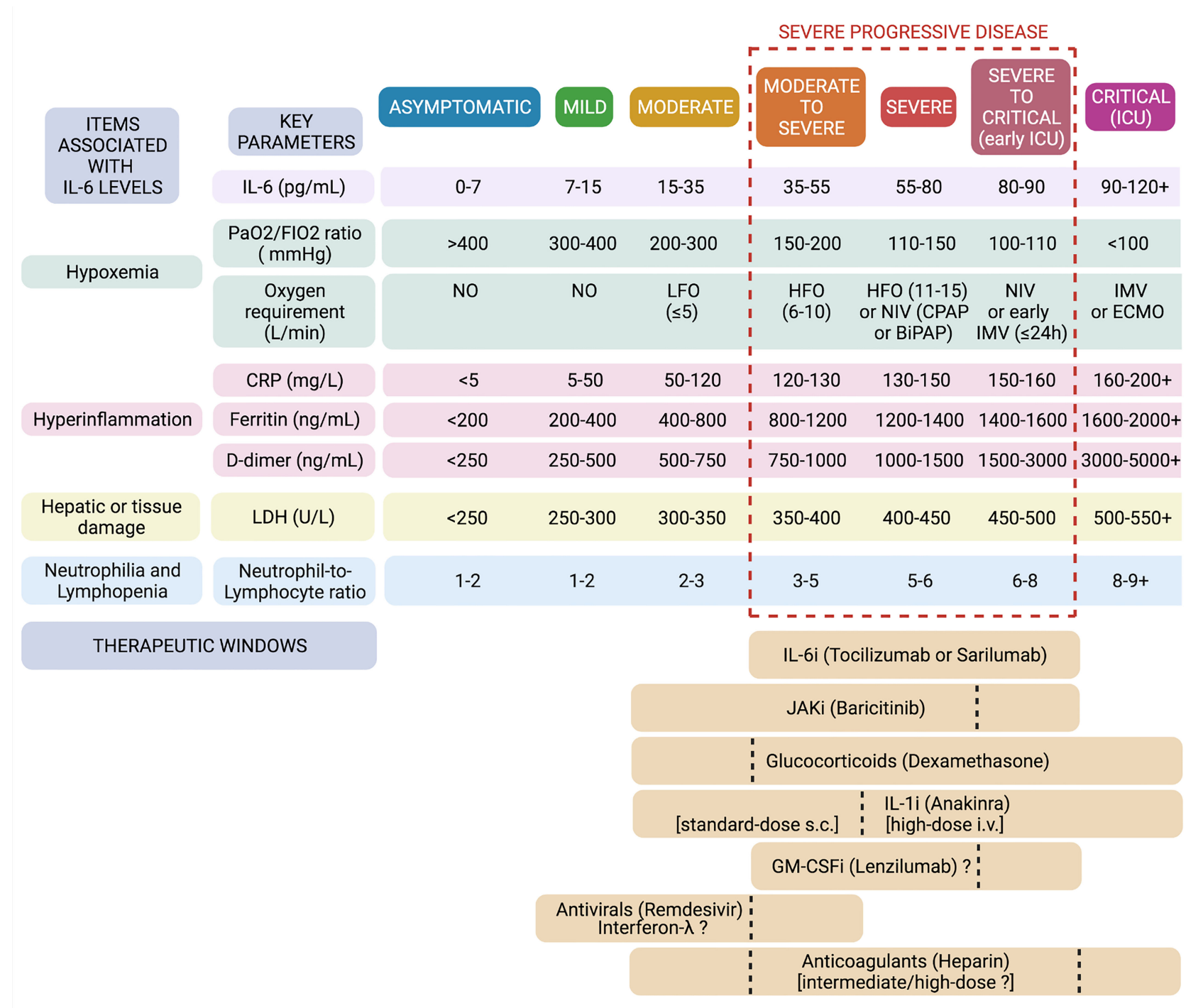

FIGURE 3 | Defining therapeutic windows for IL-6 inhibitors and other biologics in COVID-19. In the light of baseline characteristics of previous successful and unsuccessful trials, univariate and multivariate analyses, meta-analyses, and studies on the predictivity of IL-6 and other biomarkers, we set thresholds and ranges for some major respiratory and laboratory parameters in the attempt to frame specific windows of opportunity for tocilizumab and other agents in the immunotherapy of COVID-19. Created with Biorender.com.

from the conspicuous presence of monocytes-macrophages and viral products (e.g., viroporins), provokes intense production of IL- $1 \alpha$ and IL-1 $\beta$, which ultimately drive hyperinflammation and severe ARDS $(7,27)$, whereas IL-6 would now play regulatory roles. Two phase-3 RCTs evaluating the efficacy of IL-1 inhibitors in patients hospitalised with COVID-19 have recently been published, confirming significant beneficial effects on clinical status and survival for anakinra (98), but not for the anti-IL-1 $\beta$ monoclonal antibody canakinumab (114), thereby suggesting a central contribution of the alarmin IL- $1 \alpha$ to acute lung inflammation and COVID-19 pathogenesis (7).

Limited data are available from RCTs in regard to therapies targeting other cytokines involved in severe COVID-19. Among GM-CSF inhibitors, otilimab (115) conferred a benefit on survival and respiratory status in a subgroup of patients aged $\geq$ 70 years old; lenzilumab (116) improved ventilator-free survival in hypoxic patients not receiving IMV; while for mavrilimumab (117) results were inconclusive, reporting numerical but not statistical differences on mortality, possibly due to the small size and the low power of the study. It also remains to adequately investigate the effects of inhibiting IL-17, by means of anti-IL$17 \mathrm{~A} / \mathrm{F}$ therapies (e.g., bimekizumab), in severe forms, or the alarmin cytokine IL-33, by means of anti-ST2 agents (e.g., astegolimab), at least in early and moderate stages, or in postCOVID fibrosis (7).

Regarding glucocorticoids, treatment with low-to-intermediate doses of steroids, particularly dexamethasone, has been shown to be associated with reduced mortality in hypoxic patients who 
TABLE 3 | Randomised controlled trials with JAK inhibitors in COVID-19.

\begin{tabular}{|c|c|c|c|c|}
\hline PROVED EFFICACY & PARTIAL & YES & YES & YES \\
\hline \multicolumn{5}{|l|}{ TRIAL CHARACTERISTICS: } \\
\hline Authors & Cao & Kalil & Marconi & Guimaraes \\
\hline \multirow[t]{2}{*}{ [Ref.] } & et al. & et al. & et al. & et al. \\
\hline & $(107)$ & $(108)$ & $(109)$ & $(111)$ \\
\hline Study name & & ACTT-2 & COV-BARRIER & STOP-COVID \\
\hline (Geographic area) & (China) & (Multinational) & (Multinational) & (Brazil) \\
\hline Design of the study & Single-blind & Double-blind & Double-blind & Double-blind \\
\hline \multirow[t]{4}{*}{ Date of (online) publication } & May 26, & Dec 11, & Sep 1, & Jun 16, \\
\hline & 2020 & 2020 & 2021 & 2021 \\
\hline & & & (Preprint posted & \\
\hline & & & May 30, 2021) & \\
\hline \multirow[t]{2}{*}{ Period of enrollment } & Feb 9 - & May 8 - & Jun 11, 2020 - & Sep 16 - \\
\hline & 28,2020 & Jul 1, 2020 & Jan 15, 2021 & Dec 13, 2020 \\
\hline $\begin{array}{l}\text { Modified intention-to-treat } \\
\text { population }\end{array}$ & 41 & 1033 & 1525 & 289 \\
\hline $\begin{array}{l}\text { N. Patients in the treatment } \\
\text { arm }\end{array}$ & 21 & 515 & 764 & 144 \\
\hline Treatment arm & Ruxolitinib 5 mg bid & $\begin{array}{l}\text { Baricitinib } 4 \text { mg per day for } 14 \\
\text { days }\end{array}$ & $\begin{array}{l}\text { Baricitinib } 4 \text { mg per day for } \\
14 \text { days }\end{array}$ & $\begin{array}{l}\text { Tofacitinib } 10 \mathrm{mg} \text { bid for } 14 \\
\text { days }\end{array}$ \\
\hline \multicolumn{5}{|c|}{ PATIENT CHARACTERISTICS (treatment arm): } \\
\hline (Median) Age (years old) & 63 & (mean) 55 & (mean) 58 & (mean) 55 \\
\hline Male sex (\%) & 60 & 62 & 64 & 65 \\
\hline \multirow[t]{2}{*}{ Race/Ethnicity (\%) } & Asian 100 & White 49 (Hispanic 25), & White 64 (Hispanic 21), & White 82, \\
\hline & & $\begin{array}{l}\text { Black 15, Asian 9.5, Other/ } \\
\text { Unknown } 27\end{array}$ & Black 5, Asian 11, Other 20 & $\begin{array}{l}\text { Black 8, Mixed 8, Other/ } \\
\text { Unknown } 2\end{array}$ \\
\hline (Median) BMl & & (mean) 32 & (mean) 30 & 29 \\
\hline Type-2 Diabetes (\%) & 25 & & 29 & 24 \\
\hline Hypertension (\%) & 35 & & 48 & 46.5 \\
\hline N. Days from hospitalization & & & & 5 \\
\hline N. Days from symptom onset & & 8 & (mean) $\geq 7$ days in $82 \%$ & 10 \\
\hline Glucocorticoids (\%) & 70 & 22.5 & 80 & 79 \\
\hline Remdesivir (\%) & 0 & 100 & 18 & 0 \\
\hline Ambient air (\%) & 0 & 13.5 & 12 & 24 \\
\hline LFO (\%) & 90 & 56 & 64 & 63 \\
\hline HFO or NIV/cPAP (\%) & 10 & 20 & 24 & 13 \\
\hline IMV or ECMO (\%) & 0 & 10.5 & 0 & 0 \\
\hline
\end{tabular}

\section{OUTCOMES (treatment vs. standard-of-care):}

\section{Clinical status or (14-day) 60 vs 43, p=NS;}

Functional Improvement (\%) Time to improvement: 12 vs 15 days, $\mathrm{p}=\mathrm{NS}$ $\begin{array}{ll}\text { (28-day) Discharge (\%) } & \text { Time to discharge: } 17 \text { vs } 16 \text { days, } \\ \mathrm{p}=\mathrm{NS}\end{array}$

(28-day) Progression (\%)

\section{(14-day) Clinical deterioration: 0 vs $19, p=N S$; \\ (14-day) CT improvement: \\ 90 vs 62 , RR 1.45, $p=0.0495$}

(28-day) IMV (\%)

(28-day) IMV or death (\%)

(28-day) Mortality (\%)

Serious adverse events (\%) Serious infections (\%)

Venous thromboembolism (\%) Effects on virus clearance

\section{0 vs $14, p=N S$;}

cumulative incidence of death: RR 0.15, $\mathrm{p}=\mathbf{0 . 0 8 9}$

0 vs 19 0 vs 5
No)

\section{(15-day) OR 1.3}

(HFO/NIV: OR 2.2)

Median time to recovery:

7 vs 8 days, RR 1.16, $p=0.03$ (HFO/NIV: 10 vs 18 days, RR 1.51)

New use of oxygen:

23 vs 40 , HR 0.53

10 vs 15 , HR 0.64 ; IMV duration: 16 vs 27 days HR 0.69

5 vs 8 , HR 0.65 ;

(LFO: 2 vs 5, HR 0.40;

HFO/NIV: 7.5 vs 13, HR 0.55)

16 vs $21, p=0.03$

6 vs $11, p=0.003$

(concomitant steroids: 25 vs

5.5)

4 vs 3

Score worsening: OR 0.54

93 vs 89 (OR 1.05)

28 vs $30.5, p=N S$, OR 0.85

Respiratory failure or death:

18 vs 29 , OR $0.63, p=0.04$

No impairment in IFN $\alpha$ production; time to virus clearance 13 vs 12 days, $p=N S$; time to lymphocyte recovery: 5 vs 8 days, $\mathrm{p}=0.033$; increased virus-specific IgM titers, $\mathrm{p}=0.039$ [Cao et al. (107)]

Differences in key characteristics compared to trials with IL-6 inhibitors are highlighted. LEGEND OF COLOURS in the CHARACTERISTICS session: Green: favourable characteristics in studies with IL-6 inhibitors; Blue: nearly-favourable characteristics in studies with IL-6 inhibitors; Red: unfavourable characteristics in studies with IL-6 inhibitors (see Tables 1, 2).

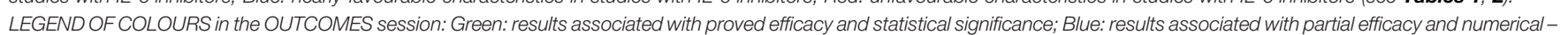
but not statistical - differences; Red: non-significant results associated with lack of efficacy or adverse events.

$H R$, hazard ratio; OR, odds ratio; $R R$, relative risk; NS, not significant. 
TABLE 4 | Randomized controlled clinical trials with agents targeting the IL-6 signalling in COVID-19 that are ongoing or awaiting results (as reported in Clinicaltrials.gov; last accessed December 6, 2021).

\begin{tabular}{|c|c|c|c|c|c|c|c|c|c|}
\hline Trial ID & Name & Phase & Masking & $\begin{array}{l}\text { Recruitment } \\
\text { Status }\end{array}$ & $\begin{array}{c}\text { Actual } \\
\text { (or Estimated) } \\
\text { Completion Date }\end{array}$ & $\begin{array}{c}\text { Actual } \\
\text { (or Estimated) } \\
\text { Number of Patients } \\
\text { Enrolled }\end{array}$ & $\begin{array}{l}\text { (Main) } \\
\text { Location }\end{array}$ & Experimental Intervention(s) & Comparator(s) \\
\hline NCT04479358 & COVIDOSE-2 & $\|$ & open-label & recruiting & March 2023 & (332) & USA & low-dose tocilizumab (40 or 120 mg) & standard-dose tocilizumab or SOC \\
\hline NCT04412772 & ARCHITECTS & III & $\begin{array}{l}\text { double- } \\
\text { blind }\end{array}$ & recruiting & December 2021 & (300) & USA & tocilizumab & placebo \\
\hline NCT04377750 & & IV & open-label & recruiting & (May 2021) & $(500)$ & Israel & tocilizumab & placebo \\
\hline NCT05002517 & & III & triple-blind & $\begin{array}{l}\text { active, not } \\
\text { recruiting }\end{array}$ & (October 2021) & 60 & Spain & tocilizumab & methylprednisolone \\
\hline NCT04519385 & & N/A & $\begin{array}{l}\text { double- } \\
\text { blind }\end{array}$ & completed & August 2020 & 69 & Egypt & tocilizumab & dexamethasone \\
\hline NCT04476979 & TOCIDEX & $\|$ & open-label & recruiting & December 2021 & (660) & $\begin{array}{l}\text { French } \\
\text { Guiana }\end{array}$ & tozilizumab + dexamethasone & dexamethasone \\
\hline NCT04577534 & COVIDSTORM & III & open-label & completed & June 2021 & 88 & Finland & tocilizumab & $\mathrm{SOC}$ \\
\hline NCT04690920 & & N/A & open-label & completed & December 2020 & 200 & Pakistan & tocilizumab or remdesivir & $\mathrm{sOC}$ \\
\hline NCT04412291 & ImmCoVA & $\|$ & open-label & recruiting & (June 2021) & $(120)$ & Sweden & tocilizumab or anakinra (+ SOC) & SOC \\
\hline NCT04678739 & & III & open-label & completed & February 2021 & 205 & Bangladesh & tozilizumab + remdesivir & SOC \\
\hline NCT04409262 & REMDACTA & III & $\begin{array}{l}\text { double- } \\
\text { blind }\end{array}$ & completed & March 2021 & 649 & USA & tocilizumab + remdesivir & placebo + remdesivir \\
\hline NCT04779047 & & IV & open-label & recruiting & (April 2021) & $(150)$ & Egypt & $\begin{array}{l}\text { tocilizumab + remdesivir } \\
\text { + lopinavir/ritonavir }\end{array}$ & $\begin{array}{l}\text { tocilizumab + ivermectin } \\
\text { + hydroxychloroquine }\end{array}$ \\
\hline NCT04310228 & & N/A & open-label & recruiting & (May 2020) & (150) & China & tocilizumab + favipiravir & tocilizumab or favipiravir \\
\hline NCT04424056 & & III & open-label & $\begin{array}{l}\text { not yet } \\
\text { recruiting }\end{array}$ & November 2022 & (216) & France & tocilizumab or anakinra \pm ruxolitinib & sOC \\
\hline NCT04330638 & COV-AID & III & open-label & completed & April 2021 & 342 & Belgium & $\begin{array}{l}\text { tocilizumab or siltuximab or anakinra } \\
\text { or tocilizumab + anakinra } \\
\text { or siltuximab + anakinra }\end{array}$ & $\mathrm{sOC}$ \\
\hline NCT04600141 & HEPMAB & III & open-label & recruiting & December 2021 & (308) & Brazil & $\begin{array}{l}\text { tocilizumab + heparin } \\
\text { (therapeutic or prophylactic } \\
\text { anticoagulation } \\
\text { with UFH or LMWH) }\end{array}$ & $\begin{array}{l}\text { heparin } \\
\text { (therapeutic or prophylactic, } \\
\text { UFH or LMWH) }\end{array}$ \\
\hline NCT05118737 & & 1 & open-label & recruiting & August 2022 & (230) & Qatar & tocilizumab + colchicine & tocilizumab \\
\hline NCT04335305 & COPERNICO & $\|$ & open-label & recruiting & (June 2021) & $(24)$ & Spain & tocilizumab + pembrolizumab & SOC \\
\hline NCT04347031 & & $\|/\|$ & open-label & completed & November 2020 & 320 & Russia & $\begin{array}{l}\text { mefloquine or hydroxychloroquine } \\
+ \text { azithromycin } \pm \text { tocilizumab }\end{array}$ & mefloquine or hydroxychloroquine \\
\hline NCT04346693 & & III & open-label & completed & November 2020 & 320 & Russia & $\begin{array}{l}\text { leitragin (intramuscular or inhaled) } \\
+ \text { hydroxychloroquine + azithromycin } \pm \\
\text { tocilizumab }\end{array}$ & $\begin{array}{l}\text { hydroxychloroquine + azithromycin } \\
\pm \text { tocilizumab }\end{array}$ \\
\hline NCT04357860 & SARICOR & $\|$ & open-label & completed & April 2021 & 120 & Spain & sarilumab sc (200 or 400 mg) (+ SOC) & $\mathrm{soc}$ \\
\hline NCT04357808 & SARCOVID & $\|$ & open-label & completed & December 2020 & 30 & Spain & sarilumab sc (+ SOC) & $\mathrm{sOC}$ \\
\hline NCT04359901 & & $\|$ & open-label & $\begin{array}{l}\text { active, not } \\
\text { recruiting }\end{array}$ & April 2023 & 50 & USA & sarilumab sc (+ SOC) & $\mathrm{SOC}$ \\
\hline NCT04324073 & $\begin{array}{l}\text { CORIMUNO- } \\
\text { SARI }\end{array}$ & $\|/\|$ & open-label & $\begin{array}{l}\text { active, not } \\
\text { recruiting }\end{array}$ & December 2021 & 239 & France & sarilumab & $\mathrm{sOC}$ \\
\hline NCT04329650 & & $\|$ & open-label & recruiting & (May 2020) & (200) & Spain & siltuximab & methylprednisolone \\
\hline NCT04343989 & & $\|$ & $\begin{array}{l}\text { double- } \\
\text { blind }\end{array}$ & completed & March 2021 & 180 & USA & clazakizumab (25 mg or $12.5 \mathrm{mg}$ ) & placebo \\
\hline NCT04363502 & & $\|$ & triple-blind & recruiting & May 2022 & (30) & USA & clazakizumab & placebo \\
\hline
\end{tabular}




\begin{tabular}{|c|c|c|c|c|c|c|c|c|c|}
\hline Trial ID & Name & Phase & Masking & $\begin{array}{l}\text { Recruitment } \\
\text { Status }\end{array}$ & $\begin{array}{c}\text { Actual } \\
\text { (or Estimated) } \\
\text { Completion Date }\end{array}$ & $\begin{array}{c}\text { Actual } \\
\text { (or Estimated) } \\
\text { Number of Patients } \\
\text { Enrolled }\end{array}$ & $\begin{array}{l}\text { (Main) } \\
\text { Location }\end{array}$ & Experimental Intervention(s) & Comparator(s) \\
\hline NCT04494724 & & $\|$ & $\begin{array}{l}\text { quadruple- } \\
\text { blind }\end{array}$ & recruiting & (July 2021) & (60) & USA & clazakizumab & placebo \\
\hline NCT04380961 & & $\|$ & $\begin{array}{l}\text { quadruple- } \\
\text { blind }\end{array}$ & completed & June 2021 & 212 & USA & sirukumab $(+\mathrm{SOC})$ & placebo (+ SOC) \\
\hline NCT04380519 & & $\|/\| \|$ & $\begin{array}{l}\text { double- } \\
\text { blind }\end{array}$ & completed & July 2020 & 372 & Russia & $\begin{array}{l}\text { olokizumab or RPH-104/goflikicept (+ } \\
\text { SOC) }\end{array}$ & placebo (+ SOC) \\
\hline NCT05056558 & & III & triple-blind & $\begin{array}{l}\text { not yet } \\
\text { recruiting }\end{array}$ & September 2022 & $(480)$ & Bangladesh & baricitinib (+ SOC) & placebo (+ SOC) \\
\hline NCT04891133 & EU SolidAct & $\|/\| I \|$ & $\begin{array}{l}\text { quadruple- } \\
\text { blind }\end{array}$ & recruiting & September 2025 & $(1900)$ & Austria & baricitinib (+ SOC) & placebo (+ SOC) \\
\hline NCT04346147 & $\begin{array}{l}\text { Covid19 } \\
\text { CovINIB }\end{array}$ & $\|$ & open-label & $\begin{array}{l}\text { active, not } \\
\text { recruiting }\end{array}$ & (September 2021) & 168 & Spain & baricitinib or imatinib & supportive treatment \\
\hline NCT04390464 & TACTIC-R & IV & open-label & recruiting & May 2022 & (1167) & UK & baricitinib or ravulizumab (+ SOC) & $\mathrm{SOC}$ \\
\hline NCT04970719 & & III & open-label & recruiting & December 2021 & (382) & Bangladesh & baricitinib + remdesivir & dexamethasone + remdesivir \\
\hline NCT04832880 & AMMURAVID & III & open-label & $\begin{array}{l}\text { not yet } \\
\text { recruiting }\end{array}$ & December 2022 & $(4000)$ & Italy & $\begin{array}{l}\text { baricitinib + remdesivir + } \\
\text { dexamethasone } \\
\text { or baricitinib + dexamethasone } \\
\text { or remdesivir + dexamethasone }\end{array}$ & dexamethasone \\
\hline NCT04640168 & ACTT-4 & III & $\begin{array}{l}\text { double- } \\
\text { blind }\end{array}$ & completed & June 2021 & 4074 & USA & baricitinib + remdesivir + placebo & $\begin{array}{l}\text { placebo + remdesivir + } \\
\text { dexamethasone }\end{array}$ \\
\hline NCT04403243 & COLORIT & $\|$ & open-label & recruiting & (August 2020) & (70) & Russia & ruxolitinib or colchicine or secukinumab & soc \\
\hline NCT04581954 & MATIS & 1/II & single-blind & recruiting & December 2021 & (456) & UK & ruxolitinib or fostamatinib & $\mathrm{SOC}$ \\
\hline NCT04348695 & Ruxo-Sim-20 & ॥ & open-label & recruiting & (May 2020) & (94) & Spain & ruxolitinib + simvastatin & SOC \\
\hline NCT05082714 & & $\mathrm{N} / \mathrm{A}$ & open-label & recruiting & April 2022 & (164) & Greece & tocilizumab & baricitinib \\
\hline NCT04693026 & & III & open-label & recruiting & (March 2021) & (150) & Bangladesh & tocilizumab + remdesivir & baricitinib + remdesivir \\
\hline
\end{tabular}

SOC, standard-of-care; UFH, unfractionated heparin; LMWH, low-molecular-weight heparin.

Bold text indicates completed studies awaiting results with a relevant number of participants or testing additional IL-6 inhibitors, interesting combinations or comparations. 
require either IMV or supplemental oxygen, but not in those who do not require oxygen support $(57,80)$. Although the main RCT in this regard (57) did not distinguish between high-flow and lowflow oxygen requirements, other studies have reported nonsignificant effects of steroids in non-severe patients requiring only low-flow oxygen $(118,119)$ (Figure 3). In case of further disease progression despite glucocorticoid treatment, the addition of tocilizumab led to significantly better outcomes $(120,121)$, consistent with the synergistic effects of tocilizumab and dexametasone observed in the RCTs and meta-analyses discussed above $(2,58)$, and possibly explained by the synergism of IL-6R signal blockade combined with steroid-induced downregulation of IL-6 (or other cytokines).

Since patients with COVID-19 at high risk of progression are characterised by early interferonopathy and viral replication (122), recombinant type-I (IFN $\beta$ ) and especially type-III (IFN $\lambda$ ) interferons, as well as endosomal TLR agonists inducing IFNs, have been proposed in patients with mildmoderate disease and high viral load (7, 123-125), similarly to what was suggested in regard to antivirals (remdesivir) (126). Nevertheless, it has been argued that antivirals might help even in more advanced stages of the disease in combination with antiinflammatory drugs $(81,126)$ (Figure 3).

COVID-19 is also characterised by acquired thrombophilia, and hypercoagulability might concur with hyperinflammation as a cause of death. Increased rates of venous thromboembolism have been observed in COVID-19 patients and autopsy studies demonstrated microvascular thrombosis in about $80 \%$ of cases (127). Furthermore, heparan sulfate may mediate virus interaction with host membrane receptors, thus promoting virus entry and inflammatory cell activation $(7,128)$. Heparin has been included in the treatment of COVID-19 with the double rationale of impeding clot formation and competing with membrane heparan sulfate. On the other side, hospitalisation could contribute to the observed risk of venous thromboembolism and the actual impact of pulmonary embolism and/or microvascular thrombosis on severe respiratory failure and mortality in COVID19 is still controversial (127). Intensified anticoagulant strategies can halve the risk of venous thromboembolism but can also double the risk of bleeding (76), especially in critically-ill patients, possibly due to tissue hypoperfusion, concomitant supportive therapy with high-dose hydrocortisone, liver failure and coagulopathy. The question of whether to prefer anticoagulant therapy or prophylaxis is therefore the subject of intense debate. Plasma concentrations of $\mathrm{D}$-dimer might be used to guide anticoagulation dose in hospitalised patients (97). Presumably, D-dimer levels < 750-1000 ng/mL (moderate disease) may suggest a low-dose prophylaxis; levels between 1000 and $3000 \mathrm{ng} / \mathrm{mL}$ (severe disease) may suggest intermediate- or high-dose therapeutic regimens; while levels > 3000-5000 ng/mL (critical disease) may warn of the risk of bleeding with intensified strategies (Figure 3). In fact, a recent meta-analysis seems to show that a full-dose anticoagulation may reduce mortality in severe, yet non-critical, patients (76).

Further data from ongoing or recently completed clinical trials are eagerly awaited (Table 4). In this regard, it will be particularly interesting to assess the effect of combining IL-6 inhibitors with antivirals (e.g., tocilizumab + remdesivir, tocilizumab + favipiravir), anticoagulants (e.g., tocilizumab + full-dose heparin), IL-1 inhibitors (e.g., tocilizumab + anakinra, tocilizumab + colchicine), or other immunotherapies (e.g., tocilizumab + pembrolizumab). Ongoing head-to-head comparative studies (e.g., tocilizumab \pm remdesivir versus baricitinib \pm remdesivir) are of great interest as well.

\section{DISCUSSION AND CONCLUSIONS}

Severe COVID-19 is a hyperinflammatory and life-threatening pulmonary and systemic disease, so prompt intervention on the cytokine cascade can importantly prevent clinical deterioration and mortality.

The beneficial effects of tocilizumab and other biologics in the management of COVID-19 have long been debated owing to large discrepancies in study results, possibly due to differences in sample size (i.e., studies often underpowered to detect significant differences in mortality), patient series composition (i.e., disease severity, magnitude of systemic inflammation, age, comorbidities, and perhaps sex and ethnicity), and treatment protocols (i.e., dosage, timing and co-treatments, particularly with steroids).

Here, we attempted to dissect the heterogeneity of these studies as well as the complexity of the inflammatory cascade in COVID-19, the differential roles of IL-6 in relation to disease stage and severity, and the biological significance of clinical parameters and laboratory markers, in order to identify and summarize the baseline characteristics of patients who should best respond to treatment with IL-6 inhibitors or other biologics, thus ultimately defining precise therapeutic windows.

Therapeutic approaches for COVID-19 based on disease stage have been suggested by subgroup analyses of clinical trials and in part summarized in previous editorials (129). Our review extends therapeutic options to anti-cytokine monoclonal antibodies and decoy receptors, such as IL-6, GM-CSF, and IL-1 inhibitors, in addition to JAK inhibitors, antivirals and glucocorticoids, with the ultimate aim of highlighting their differential windows of opportunity. Importantly, our estimation of disease severity is based not only on respiratory status, here assessed by means of the $\mathrm{PaO} 2 / \mathrm{FIO} 2$ ratio and oxygen requirement, but also on key circulating biomarkers, namely IL-6, CRP, ferritin, D-dimer and LDH levels. Furthermore, here we have distinguished a "severeto-critical” stage corresponding to early ICU patients, including those undergoing IMV within the past 24 hours (i.e., ordinal score between 6 and 7 on the NIAID 8-point ordinal scale for assessing clinical status), for whom - so far - there is evidence of benefit for IL-6 inhibitors, but not for JAK inhibitors.

In summary, due to the pathogenic role of IL- 6 signal (transsignalling and trans-presentation) specifically in the severe stage of the disease, IL-6 inhibitors, and particularly anti-IL-6R monoclonal antibodies (e.g., tocilizumab, sarilumab), appear to be effective in patients with severe COVID-19, mostly characterised by baseline IL- 6 levels between 35 and $90 \mathrm{ng} / \mathrm{mL}$ (typically reached within 6 days of hospitalisation), $\mathrm{PaO} 2 / \mathrm{FIO} 2$ 
ratios between 100 and $200 \mathrm{mmHg}$, requirement of HFO or NIV, CRP levels between 120 and $160 \mathrm{mg} / \mathrm{L}$, ferritin levels between 800 and $1600 \mathrm{ng} / \mathrm{mL}, \mathrm{D}$-dimer levels between 750 and $3000 \mathrm{ng} / \mathrm{mL}$, and LDH levels between 350 and $500 \mathrm{U} / \mathrm{L}$. Patients aged between 59 and 64 years old, males, and non-Hispanic White, might respond better than others to IL- 6 inhibitors.

Because GM-CSF also plays an important role in severe disease, GM-CSF inhibitors (e.g., lenzilumab, otilimab, mavrilimumab) may have a therapeutic window similar to that of IL-6 inhibitors, perhaps with a difference in age preference (i.e., better outcomes obtained for patients aged $\leq 70$ years old with tocilizumab, $\geq 70$ years old with otilimab).

Since JAK1 and JAK2 transmit both IL-6 and GM-CSF signals, as well as the signal of various cytokines placed aside or upstream in the inflammatory cascade (e.g., IL-9, IFN $\gamma$, IL-7, IL-23, G-CSF), JAK inhibitors, and particularly JAK1/JAK2 inhibitors (e.g., baricitinib, which might also directly interfere with viral replication), appear to be effective not only in severe forms but also in moderate COVID-19 (thus including younger patients and more women), and even in the absence of concomitant steroids.

\section{REFERENCES}

1. Available at: https://www.google.com/search?q=covid-19+cases.

2. Ghosn L, Chaimani A, Evrenoglou T, Davidson M, Graña C, Schmucker C, et al. Interleukin-6 Blocking Agents for Treating COVID-19: A Living Systematic Review. Cochrane Database Syst Rev (2021) 3(3):CD013881. doi: 10.1002/14651858.CD013881

3. Guan WJ, Ni ZY, Hu Y, Liang WH, Ou CQ, He JX, et al. Clinical Characteristics of Coronavirus Disease 2019 in China. N Engl J Med (2020) 382(18):1708-20. doi: 10.1056/NEJMoa2002032

4. Wiersinga WJ, Rhodes A, Cheng AC, Peacock SJ, Prescott HC. Pathophysiology, Transmission, Diagnosis, and Treatment of Coronavirus Disease 2019 (COVID-19): A Review. JAMA (2020) 324(8):782-93. doi: 10.1001/jama.2020.12839

5. Wu Z, McGoogan JM. Characteristics of and Important Lessons From the Coronavirus Disease 2019 (COVID-19) Outbreak in China: Summary of a Report of 72314 Cases From the Chinese Center for Disease Control and Prevention. JAMA (2020) 323(13):1239-42. doi: 10.1001 /jama.2020.2648

6. Stokes EK, Zambrano LD, Anderson KN, Marder EP, Raz KM, El Burai Felix S, et al. Coronavirus Disease 2019 Case Surveillance - United States, January 22-May 30, 2020. MMWR Morb Mortal Wkly Rep (2020) 69(24):759-65. doi: 10.15585/mmwr.mm6924e2

7. Zizzo G, Cohen PL. Imperfect Storm: Is Interleukin-33 the Achilles Heel of COVID-19? Lancet Rheumatol (2020) 2(12):e779-90. doi: 10.1016/S26659913(20)30340-4

8. Buszko M, Nita-Lazar A, Park JH, Schwartzberg PL, Verthelyi D, Young $\mathrm{HA}$, et al. Lessons Learned: New Insights on the Role of Cytokines in COVID-19. Nat Immunol (2021) 22(4):404-11. doi: 10.1038/s41590-02100901-9

9. Morena V, Milazzo L, Oreni L, Bestetti G, Fossali T, Bassoli C, et al. OffLabel Use of Tocilizumab for the Treatment of SARS-CoV-2 Pneumonia in Milan, Italy. Eur J Intern Med (2020) 76:36-42. doi: 10.1016/j.ejim. 2020.05.011

10. Richardson S, Hirsch JS, Narasimhan M, Crawford JM, McGinn T, Davidson KW, et al. Presenting Characteristics, Comorbidities, and Outcomes Among 5700 Patients Hospitalized With COVID-19 in the New York City Area. JAMA (2020) 323(20):2052-9. doi: 10.1001/jama.2020.6775

11. Tan M, Liu Y, Zhou R, Deng X, Li F, Liang K, et al. Immunopathological Characteristics of Coronavirus Disease 2019 Cases in Guangzhou, China. Immunology (2020) 160(3):261-8. doi: 10.1111/imm.13223
Whereas IL-6 acquires homeostatic roles in critical stages (cis-signalling), IL- $1 \alpha$ and IL- $1 \beta$ are instead proinflammatory and pathogenic in both severe and critical disease, being increasingly released with severe hyperferritinemia and hyperactivation of monocytes-macrophages; IL-1 inhibitors, in fact, and particularly the decoy IL-1 receptor binding to both IL1 isoforms (e.g., anakinra), appear to be effective not only in moderate-to-severe forms but also in critical COVID-19.

It is hoped that this narrative overview of the current literature may offer useful insights into the proper use of biologics in COVID-19, in future trials as in the real world.

\section{AUTHOR CONTRIBUTIONS}

GZ conceived the study, searched the literature, wrote the manuscript, and prepared the figures and tables. AT, LC, and AL contributed to search the literature and reviewed the manuscript. NM, PF, IS, and AM critically edited the manuscript. All authors have read and approved the final version submitted.

12. Liao M, Liu Y, Yuan J, Wen Y, Xu G, Zhao J, et al. Single-Cell Landscape of Bronchoalveolar Immune Cells in Patients With COVID-19. Nat Med (2020) 26(6):842-4. doi: 10.1038/s41591-020-0901-9

13. Han H, Ma Q, Li C, Liu R, Zhao L, Wang W, et al. Profiling Serum Cytokines in COVID-19 Patients Reveals IL-6 and IL-10 Are Disease Severity Predictors. Emerg Microbes Infect (2020) 9(1):1123-30. doi: 10.1080/ 22221751.2020.1770129

14. Huang C, Wang Y, Li X, Ren L, Zhao J, Hu Y, et al. Clinical Features of Patients Infected With 2019 Novel Coronavirus in Wuhan, China. Lancet (2020) 395(10223):497-506. doi: 10.1016/S0140-6736(20)30183-5

15. Chu H, Chan JF, Wang Y, Yuen TT, Chai Y, Hou Y, et al. Comparative Replication and Immune Activation Profiles of SARS-CoV-2 and SARS$\mathrm{CoV}$ in Human Lungs: An Ex Vivo Study With Implications for the Pathogenesis of COVID-19. Clin Infect Dis (2020) 71:1400-9. doi: $10.1093 / \mathrm{cid} / \mathrm{ciaa} 410$

16. Blanco-Melo D, Nilsson-Payant BE, Liu WC, Uhl S, Hoagland D, Møller R, et al. Imbalanced Host Response to SARS-CoV-2 Drives Development of COVID-19. Cell (2020) 181:1036-1045.e9. doi: 10.1016/j.cell.2020.04.026

17. Zhou Y, Fu B, Zheng X, Wang D, Zhao C, Qi Y, et al. Pathogenic T Cells and Inflammatory Monocytes Incite Inflammatory Storm in Severe COVID-19 Patients. Natl Sci Rev (2020) 7:998-1002. doi: 10.1093/nsr/nwaa041

18. Cifaldi L, Prencipe G, Caiello I, Bracaglia C, Locatelli F, De Benedetti F, et al. Inhibition of Natural Killer Cell Cytotoxicity by Interleukin-6: Implications for the Pathogenesis of Macrophage Activation Syndrome. Arthritis Rheumatol (2015) 67:3037-46. doi: 10.1002/art.39295

19. McGonagle D, Sharif K, O'Regan A, Bridgewood C. The Role of Cytokines Including Interleukin-6 in COVID-19 Induced Pneumonia and Macrophage Activation Syndrome-Like Disease. Autoimmun Rev (2020) 19:102537. doi: 10.1016/j.autrev.2020.102537

20. Chen X, Zhao B, Qu Y, Chen Y, Xiong J, Feng Y, et al. Detectable Serum Severe Acute Respiratory Syndrome Coronavirus 2 Viral Load (RNAemia) Is Closely Correlated With Drastically Elevated Interleukin 6 Level in Critically Ill Patients With Coronavirus Disease 2019. Clin Infect Dis (2020) 71 (8):1937-42. doi: 10.1093/cid/ciaa449

21. Wang F, Qu M, Zhou X, Zhao K, Lai C, Tang Q, et al. The Timeline and Risk Factors of Clinical Progression of COVID-19 in Shenzhen, China. J Transl Med (2020) 18(1):270. doi: 10.1186/s12967-020-02423-8

22. Tleyjeh IM, Kashour Z, Damlaj M, Riaz M, Tlayjeh H, Altannir M, et al. Efficacy and Safety of Tocilizumab in COVID-19 Patients: A Living Systematic Review and Meta-Analysis. Clin Microbiol Infect (2021) 27 (2):215-27. doi: 10.1016/j.cmi.2020.10.036 
23. Santa Cruz A, Mendes-Frias A, Oliveira AI, Dias L, Matos AR, Carvalho A, et al. Interleukin-6 Is a Biomarker for the Development of Fatal Severe Acute Respiratory Syndrome Coronavirus 2 Pneumonia. Front Immunol (2021) 12:613422. doi: 10.3389/fimmu.2021.613422

24. Bovijn J, Lindgren CM, Holmes MV. Genetic Variants Mimicking Therapeutic Inhibition of IL-6 Receptor Signaling and Risk of COVID-19. Lancet Rheumatol (2020) 2(11):e658-9. doi: 10.1016/S2665-9913(20)30345-3

25. Garbers C, Heink S, Korn T, Rose-John S. Interleukin-6: Designing Specific Therapeutics for a Complex Cytokine. Nat Rev Drug Discovery (2018) 17 (6):395-412. doi: 10.1038/nrd.2018.45

26. Narazaki M, Kishimoto T. The Two-Faced Cytokine IL-6 in Host Defense and Diseases. Int J Mol Sci (2018) 19(11):3528. doi: 10.3390/ijms19113528

27. Nasonov E, Samsonov M. The Role of Interleukin 6 Inhibitors in Therapy of Severe COVID-19. BioMed Pharmacother (2020) 131:110698. doi: 10.1016/ j.biopha.2020.110698

28. Mazzone A, Castelnovo L, Tamburello A, Gatti A, Brando B, Faggioli P, et al. Monocytes Could Be a Bridge From Inflammation to Thrombosis on COVID-19 Injury: A Case Report. Thromb Update (2020) 1:100007. doi: 10.1016/j.tru.2020.100007

29. Gatti A, Radrizzani D, Viganò P, Mazzone A, Brando B. Decrease of NonClassical and Intermediate Monocyte Subsets in Severe Acute SARS-CoV-2 Infection. Cytometry A. (2020) 97(9):887-90. doi: 10.1002/cyto.a.24188

30. Xu Z, Shi L, Wang Y, Zhang J, Huang L, Zhang C, et al. Pathological Findings of COVID-19 Associated With Acute Respiratory Distress Syndrome. Lancet Respir Med (2020) 8:420-2. doi: 10.1016/S2213-2600(20)30076-X

31. Petes C, Mariani MK, Yang Y, Grandvaux N, Gee K. Interleukin (IL)-6 Inhibits IL-27- and IL-30-Mediated Inflammatory Responses in Human Monocytes. Front Immunol (2018) 9:256. doi: 10.3389/fimmu.2018.00256

32. WHO Rapid Evidence Appraisal for COVID-19 Therapies (REACT) Working Group, Shankar-Hari M, Vale CL, Godolphin PJ, Fisher D, Higgins JPT, et al. Association Between Administration of IL-6 Antagonists and Mortality Among Patients Hospitalized for COVID-19: A Meta-Analysis. JAMA (2021) 326(6):499-518. doi: 10.1001/jama.2021.11330

33. Aderka D, Le JM, Vilcek J. IL-6 Inhibits Lipopolysaccharide-Induced Tumor Necrosis Factor Production in Cultured Human Monocytes, U937 Cells, and in Mice. J Immunol (1989) 143(11):3517-23.

34. Tilg H, Trehu E, Atkins MB, Dinarello CA, Mier JW. Interleukin-6 (IL-6) as an Anti-Inflammatory Cytokine: Induction of Circulating IL-1 Receptor Antagonist and Soluble Tumor Necrosis Factor Receptor P55. Blood (1994) 83(1):113-8. doi: 10.1182/blood.V83.1.113.bloodjournal831113

35. Cooney LA, Towery K, Endres J, Fox DA. Sensitivity and Resistance to Regulation by IL-4 During Th17 Maturation. J Immunol (2011) 187 (9):4440-50. doi: 10.4049/jimmunol.1002860

36. Niemand C, Nimmesgern A, Haan S, Fischer P, Schaper F, Rossaint R, et al. Activation of STAT3 by IL-6 and IL-10 in Primary Human Macrophages Is Differentially Modulated by Suppressor of Cytokine Signaling 3. J Immunol (2003) 170(6):3263-72. doi: 10.4049/jimmunol.170.6.3263

37. Kimmig LM, Wu D, Gold M, Pettit NN, Pitrak D, Mueller J, et al. IL-6 Inhibition in Critically Ill COVID-19 Patients Is Associated With Increased Secondary Infections. Front Med (Lausanne) (2020) 7:583897. doi: 10.3389/ fmed.2020.583897

38. Somers EC, Eschenauer GA, Troost JP, Golob JL, Gandhi TN, Wang L, et al. Tocilizumab for Treatment of Mechanically Ventilated Patients With COVID-19. Clin Infect Dis (2021) 73(2):e445-54. doi: 10.1093/cid/ciaa954

39. Johnson HM, Lewin AS, Ahmed CM. SOCS, Intrinsic Virulence Factors, and Treatment of COVID-19. Front Immunol (2020) 11:582102. doi: 10.3389/ fimmu.2020.582102

40. Lang R, Pauleau AL, Parganas E, Takahashi Y, Mages J, Ihle JN, et al. SOCS3 Regulates the Plasticity of Gp130 Signaling. Nat Immunol (2003) 4(6):54650. doi: 10.1038/ni932

41. Liu S, Yan R, Chen B, Pan Q, Chen Y, Hong J, et al. Influenza Virus-Induced Robust Expression of SOCS3 Contributes to Excessive Production of IL-6. Front Immunol (2019) 10:1843. doi: 10.3389/fimmu.2019.01843

42. Diehl S, Rincón M. The Two Faces of IL-6 on Th1/Th2 Differentiation. Mol Immunol (2002) 39(9):531-6. doi: 10.1016/s0161-5890(02)00210-9

43. Veiga VC, Prats JAGG, Farias DLC, Rosa RG, Dourado LK, Zampieri FG, et al. Coalition Covid-19 Brazil VI Investigators. Effect of Tocilizumab on Clinical Outcomes at 15 Days in Patients With Severe or Critical
Coronavirus Disease 2019: Randomised Controlled Trial. BMJ (2021) 372: n84. doi: 10.1136/bmj.n84

44. Choy EH, De Benedetti F, Takeuchi T, Hashizume M, John MR, Kishimoto T. Translating IL-6 Biology Into Effective Treatments. Nat Rev Rheumatol (2020) 16(6):335-45. doi: 10.1038/s41584-020-0419-Z

45. Colaneri M, Bogliolo L, Valsecchi P, Sacchi P, Zuccaro V, Brandolino F, et al. The Covid Irccs San Matteo Pavia Task Force. Tocilizumab for Treatment of Severe COVID-19 Patients: Preliminary Results From SMAtteo COvid19 REgistry (SMACORE). Microorganisms (2020) 8(5):695. doi: 10.3390/ microorganisms 8050695

46. Capra R, De Rossi N, Mattioli F, Romanelli G, Scarpazza C, Sormani MP, et al. Impact of Low Dose Tocilizumab on Mortality Rate in Patients With COVID-19 Related Pneumonia. Eur J Intern Med (2020) 76:31-5. doi: 10.1016/j.ejim.2020.05.009

47. Quartuccio L, Sonaglia A, McGonagle D, Fabris M, Peghin M, Pecori D, et al. Profiling COVID-19 Pneumonia Progressing Into the Cytokine Storm Syndrome: Results From a Single Italian Centre Study on Tocilizumab Versus Standard of Care. J Clin Virol (2020) 129:104444. doi: 10.1016/j.jcv.2020.104444

48. Campochiaro C, Della-Torre E, Cavalli G, De Luca G, Ripa M, Boffini N, et al. Efficacy and Safety of Tocilizumab in Severe COVID-19 Patients: A Single-Centre Retrospective Cohort Study. Eur J Intern Med (2020) 76:43-9. doi: 10.1016/j.ejim.2020.05.021

49. Gritti G, Raimondi F, Ripamonti D, Riva I, Landi F, Alborghetti L, et al. IL-6 Signalling Pathway Inactivation With Siltuximab in Patients With COVID19 Respiratory Failure: An Observational Cohort Study. medRxiv (2020) 04.01.20048561. doi: 10.1101/2020.04.01.20048561

50. Guaraldi G, Meschiari M, Cozzi-Lepri A, Milic J, Tonelli R, Menozzi M, et al. Tocilizumab in Patients With Severe COVID-19: A Retrospective Cohort Study. Lancet Rheumatol (2020) 2(8):e474-84. doi: 10.1016/S2665-9913(20) 30173-9

51. Della-Torre E, Campochiaro C, Cavalli G, De Luca G, Napolitano A, La Marca S, et al. Interleukin-6 Blockade With Sarilumab in Severe COVID-19 Pneumonia With Systemic Hyperinflammation: An Open-Label Cohort Study. Ann Rheum Dis (2020) 79(10):1277-85. doi: 10.1136/annrheumdis2020-218122

52. Rossotti R, Travi G, Ughi N, Corradin M, Baiguera C, Fumagalli R, et al. Safety and Efficacy of Anti-Il6-Receptor Tocilizumab Use in Severe and Critical Patients Affected by Coronavirus Disease 2019: A Comparative Analysis. J Infect (2020) 81(4):e11-7. doi: 10.1016/j.jinf.2020.07.008

53. Canziani LM, Trovati S, Brunetta E, Testa A, De Santis M, Bombardieri E, et al. Humanitas and Gavazzeni / Castelli COVID-19 Task Forces. Interleukin-6 Receptor Blocking With Intravenous Tocilizumab in COVID-19 Severe Acute Respiratory Distress Syndrome: A Retrospective Case-Control Survival Analysis of 128 Patients. J Autoimmun (2020) 114:102511. doi: 10.1016/j.jaut.2020.102511

54. Castelnovo L, Tamburello A, Lurati A, Zaccara E, Marrazza MG, Olivetti M, et al. Anti-IL6 Treatment of Serious COVID-19 Disease: A Monocentric Retrospective Experience. Med (Baltimore) (2021) 100(1):e23582. doi: 10.1097/MD.0000000000023582

55. Ghazy RM, Almaghraby A, Shaaban R, Kamal A, Beshir H, Moursi A, et al. A Systematic Review and Meta-Analysis on Chloroquine and Hydroxychloroquine as Monotherapy or Combined With Azithromycin in COVID-19 Treatment. Sci Rep (2020) 10(1):22139. doi: 10.1038/s41598020-77748-x

56. Echarte-Morales J, Minguito-Carazo C, Del Castillo-García S, BorregoRodríguez J, Rodríguez-Santamarta M, Sánchez-Muñoz E, et al. Effect of Hydroxychloroquine, Azithromycin and Lopinavir/Ritonavir on the QT Corrected Interval in Patients With COVID-19. J Electrocardiol (2021) 64:30-5. doi: 10.1016/j.jelectrocard.2020.11.012

57. Collaborative Group RECOVERY, Horby P, Lim WS, Emberson JR, Mafham M, Bell JL, et al. Dexamethasone in Hospitalized Patients With Covid-19. N Engl J Med (2021) 384(8):693-704. doi: 10.1056/NEJMoa2021436

58. RECOVERY Collaborative Group. Tocilizumab in Patients Admitted to Hospital With COVID-19 (RECOVERY): A Randomised, Controlled, Open-Label, Platform Trial. Lancet (2021) 397(10285):1637-45. doi: 10.1016/S0140-6736(21)00676-0

59. Gremese E, Cingolani A, Bosello SL, Alivernini S, Tolusso B, Perniola S, et al. Sarilumab Use in Severe SARS-CoV-2 Pneumonia. EClinicalMedicine (2020) 27:100553. doi: $10.1016 /$ j.eclinm.2020.100553 
60. Perrone F, Piccirillo MC, Ascierto PA, Salvarani C, Parrella R, Marata AM, et al. Tocilizumab for Patients With COVID-19 Pneumonia. The SingleArm TOCIVID-19 Prospective Trial. J Transl Med (2020) 18(1):405. doi: 10.1186/s12967-020-02573-9

61. Sciascia S, Aprà F, Baffa A, Baldovino S, Boaro D, Boero R, et al. Pilot Prospective Open, Single-Arm Multicentre Study on Off-Label Use of Tocilizumab in Patients With Severe COVID-19. Clin Exp Rheumatol (2020) 38(3):529-32.

62. Pomponio G, Ferrarini A, Bonifazi M, Moretti M, Salvi A, Giacometti A, et al. Tocilizumab in COVID-19 Interstitial Pneumonia. J Intern Med (2021) 289(5):738-46. doi: 10.1111/joim.13231

63. Toniati P, Piva S, Cattalini M, Garrafa E, Regola F, Castelli F, et al. Tocilizumab for the Treatment of Severe COVID-19 Pneumonia With Hyperinflammatory Syndrome and Acute Respiratory Failure: A Single Center Study of 100 Patients in Brescia, Italy. Autoimmun Rev (2020) 19 (7):102568. doi: 10.1016/j.autrev.2020.102568

64. Patel S, Saxena B, Mehta P. Recent Updates in the Clinical Trials of Therapeutic Monoclonal Antibodies Targeting Cytokine Storm for the Management of COVID-19. Heliyon (2021) 7(2):e06158. doi: 10.1016/ j.heliyon.2021.e06158

65. Investigators REMAP-CAP, Gordon AC, Mouncey PR, Al-Beidh F, Rowan KM, Nichol AD, et al. Interleukin-6 Receptor Antagonists in Critically Ill Patients With Covid-19. N Engl J Med (2021) 384(16):1491-502. doi: 10.1056/NEJMoa2100433

66. Rosas IO, Bräu N, Waters M, Go RC, Hunter BD, Bhagani S, et al. Tocilizumab in Hospitalized Patients With Severe Covid-19 Pneumonia. N Engl J Med (2021) 384(16):1503-16. doi: 10.1056/NEJMoa2028700

67. Wang D, Fu B, Peng Z, Yang D, Han M, Li M, et al. Tocilizumab in Patients With Moderate or Severe COVID-19: A Randomized, Controlled, OpenLabel, Multicenter Trial. Front Med (2021) 15(3):486-94. doi: 10.1007/ s11684-020-0824-3

68. Soin AS, Kumar K, Choudhary NS, Sharma P, Mehta Y, Kataria S, et al. Tocilizumab Plus Standard Care Versus Standard Care in Patients in India With Moderate to Severe COVID-19-Associated Cytokine Release Syndrome (COVINTOC): An Open-Label, Multicentre, Randomised, Controlled, Phase 3 Trial. Lancet Respir Med (2021) 9(5):511-21. doi: 10.1016/S2213-2600(21)00081-3

69. Lescure FX, Honda H, Fowler RA, Lazar JS, Shi G, Wung P, et al. Sarilumab in Patients Admitted to Hospital With Severe or Critical COVID-19: A Randomised, Double-Blind, Placebo-Controlled, Phase 3 Trial. Lancet Respir Med (2021) 9(5):522-32. doi: 10.1016/\$2213-2600(21)00099-0

70. Salama C, Han J, Yau L, Reiss WG, Kramer B, Neidhart JD, et al. Tocilizumab in Patients Hospitalized With Covid-19 Pneumonia. N Engl J Med (2021) 384(1):20-30. doi: 10.1056/NEJMoa2030340

71. Stone JH, Frigault MJ, Serling-Boyd NJ, Fernandes AD, Harvey L, Foulkes AS, et al. Efficacy of Tocilizumab in Patients Hospitalized With Covid-19. N Engl J Med (2020) 383(24):2333-44. doi: 10.1056/NEJMoa2028836

72. Hermine O, Mariette X, Tharaux PL, Resche-Rigon M, Porcher R, Ravaud P, et al. Effect of Tocilizumab vs Usual Care in Adults Hospitalized With COVID19 and Moderate or Severe Pneumonia: A Randomized Clinical Trial. JAMA Intern Med (2021) 181(1):32-40. doi: 10.1001/jamainternmed.2020.6820

73. Salvarani C, Dolci G, Massari M, Merlo DF, Cavuto S, Savoldi L, et al. Effect of Tocilizumab vs Standard Care on Clinical Worsening in Patients Hospitalized With COVID-19 Pneumonia: A Randomized Clinical Trial. JAMA Intern Med (2021) 181(1):24-31. doi: 10.1001/jamainternmed.2020.6615

74. Kumar PN, Hernández-Sánchez J, Nagel S, Feng Y, Cai F, Rabin J, et al. Safety and Efficacy of Tocilizumab 4 or $8 \mathrm{mg} / \mathrm{kg}$ in Hospitalized Patients With Moderate to Severe Coronavirus Disease 2019 Pneumonia: A Randomized Clinical Trial. Open Forum Infect Dis (2021) 9(1):ofab608. doi: 10.1093/ofid/ofab608

75. Lin WT, Hung SH, Lai CC, Wang CY, Chen CH. The Effect of Tocilizumab on COVID-19 Patient Mortality: A Systematic Review and Meta-Analysis of Randomized Controlled Trials. Int Immunopharmacol (2021) 96:107602. doi: 10.1016/j.intimp.2021.107602

76. Siemieniuk RA, Bartoszko JJ, Ge L, Zeraatkar D, Izcovich A, Kum E, et al. Drug Treatments for Covid-19: Living Systematic Review and Network MetaAnalysis. BMJ (2020) 370:m2980. doi: 10.1136/bmj.m2980. Update in: BMJ

77. Boregowda U, Perisetti A, Nanjappa A, Gajendran M, Kutti GSridharan, Goyal H. Addition of Tocilizumab to the Standard of Care Reduces
Mortality in Severe COVID-19: A Systematic Review and Meta-Analysis. Front Med (Lausanne) (2020) 7:586221. doi: 10.3389/fmed.2020.586221

78. Han Q, Guo M, Zheng Y, Zhang Y, De Y, Xu C, et al. Current Evidence of Interleukin-6 Signaling Inhibitors in Patients With COVID-19: A Systematic Review and Meta-Analysis. Front Pharmacol (2020) 11:615972. doi: 10.3389/ fphar.2020.615972

79. Albuquerque AM, Tramujas L, Sewanan LR, Brophy JM. Tocilizumab in COVID-19 - A Bayesian Reanalysis of RECOVERY. medRxiv preprint (2021). doi: 10.1101/2021.06.15.21258966

80. WHO Rapid Evidence Appraisal for COVID-19 Therapies (REACT) Working Group, Sterne JAC, Murthy S, Diaz JV, Slutsky AS, Villar J, et al. Association Between Administration of Systemic Corticosteroids and Mortality Among Critically Ill Patients With COVID-19: A Meta-Analysis. JAMA (2020) 324(13):1330-41. doi: 10.1001/jama.2020.17023

81. COVID-19 Treatment Guidelines Panel. Coronavirus Disease 2019 (COVID-19) Treatment Guidelines. National Institutes of Health. Available at: https://www.covid19treatmentguidelines.nih.gov/ (Accessed September 30, 2021).

82. Zhang J, Hao Y, Ou W, Ming F, Liang G, Qian Y, et al. Serum Interleukin-6 Is an Indicator for Severity in 901 Patients With SARS-CoV-2 Infection: A Cohort Study. J Transl Med (2020) 18(1):406. doi: 10.1186/s12967-02002571-x

83. Coomes EA, Haghbayan H. Interleukin-6 in Covid-19: A Systematic Review and Meta-Analysis. Rev Med Virol (2020) 30(6):1-9. doi: 10.1002/rmv.2141

84. Aziz M, Fatima R, Assaly R. Elevated Interleukin-6 and Severe COVID-19: A Meta-Analysis. J Med Virol (2020) 92(11):2283-5. doi: 10.1002/jmv.25948

85. Chen LYC, Hoiland RL, Stukas S, Wellington CL, Sekhon MS. Confronting the Controversy: Interleukin- 6 and the COVID-19 Cytokine Storm Syndrome. Eur Respir J (2020) 56(4):2003006. doi: 10.1183/13993003.03006-2020

86. Herold T, Jurinovic V, Arnreich C, Lipworth BJ, Hellmuth JC, von BergweltBaildon M, et al. Elevated Levels of IL- 6 and CRP Predict the Need for Mechanical Ventilation in COVID-19. J Allergy Clin Immunol (2020) 146 (1):128-136.e4. doi: 10.1016/j.jaci.2020.05.008

87. Laguna-Goya R, Utrero-Rico A, Talayero P, Lasa-Lazaro M, RamirezFernandez A, Naranjo L, et al. IL-6-Based Mortality Risk Model for Hospitalized Patients With COVID-19. J Allergy Clin Immunol (2020) 146 (4):799-807.e9. doi: 10.1016/j.jaci.2020.07.009

88. Tian H, Sui Y, Tian S, Zou X, Xu Z, He H, et al. Case Report: Clinical Treatment of the First Critical Patient With Coronavirus Disease (COVID19) in Liaocheng, Shandong Province. Front Med (Lausanne) (2020) 7:249. doi: $10.3389 /$ fmed.2020.00249

89. Caricchio R, Gallucci M, Dass C, Zhang X, Gallucci S, Fleece D, et al. Preliminary Predictive Criteria for COVID-19 Cytokine Storm. Ann Rheum Dis (2021) 80(1):88-95. doi: 10.1136/annrheumdis-2020-218323

90. Leisman DE, Ronner L, Pinotti R, Taylor MD, Sinha P, Calfee CS, et al. Cytokine Elevation in Severe and Critical COVID-19: A Rapid Systematic Review, Meta-Analysis, and Comparison With Other Inflammatory Syndromes. Lancet Respir Med (2020) 8(12):1233-44. doi: 10.1016/S22132600(20)30404-5

91. Gómez-Pastora J, Weigand M, Kim J, Wu X, Strayer J, Palmer AF, et al. Hyperferritinemia in Critically Ill COVID-19 Patients - Is Ferritin the Product of Inflammation or a Pathogenic Mediator? Clin Chim Acta (2020) 509:249-51. doi: 10.1016/j.cca.2020.06.033

92. Kappert K, Jahić A, Tauber R. Assessment of Serum Ferritin as a Biomarker in COVID-19: Bystander or Participant? Insights by Comparison With Other Infectious and non-Infectious Diseases. Biomarkers (2020) 25(8):61625. doi: 10.1080/1354750X.2020.1797880

93. Fardet L, Galicier L, Lambotte O, Marzac C, Aumont C, Chahwan D, et al. Development and Validation of the HScore, a Score for the Diagnosis of Reactive Hemophagocytic Syndrome. Arthritis Rheumatol (2014) 66 (9):2613-20. doi: 10.1002/art.38690

94. Davì S, Minoia F, Pistorio A, Horne A, Consolaro A, Rosina S, et al. Performance of Current Guidelines for Diagnosis of Macrophage Activation Syndrome Complicating Systemic Juvenile Idiopathic Arthritis. Arthritis Rheumatol (2014) 66(10):2871-80. doi: 10.1002/art.38769

95. Della-Torre E, Lanzillotta M, Campochiaro C, Cavalli G, De Luca G, Tomelleri A, et al. Respiratory Impairment Predicts Response to IL-1 and IL-6 Blockade in COVID-19 Patients With Severe Pneumonia and Hyper- 
Inflammation. Front Immunol (2021) 12:675678. doi: 10.3389/ fimmu.2021.675678

96. Zhou F, Yu T, Du R, Fan G, Liu Y, Liu Z, et al. Clinical Course and Risk Factors for Mortality of Adult Inpatients With COVID-19 in Wuhan, China: A Retrospective Cohort Study. Lancet (2020) 395(10229):1054-62. doi: 10.1016/S0140-6736(20)30566-3

97. Conte G, Cei M, Evangelista I, Colombo A, Vitale J, Mazzone A, et al. The Meaning of D-Dimer Value in Covid-19. Clin Appl Thromb Hemost (2021) 27:10760296211017668. doi: 10.1177/10760296211017668

98. Kyriazopoulou E, Poulakou G, Milionis H, Metallidis S, Adamis G, Tsiakos K, et al. Early Treatment of COVID-19 With Anakinra Guided by Soluble Urokinase Plasminogen Receptor Plasma Levels: A Double-Blind, Randomized Controlled Phase 3 Trial. Nat Med (2021) 27(10):1752-60. doi: 10.1038/s41591-021-01499-z

99. Sivapalasingam S, Lederer DJ, Bhore R, Hajizadeh N, Criner G, Hosain R, et al. A Randomized Placebo-Controlled Trial of Sarilumab in Hospitalized Patients With Covid-19. medRxiv preprint (2021). doi: 10.1101/ 2021.05.13.21256973

100. Stebbing J, Phelan A, Griffin I, Tucker C, Oechsle O, Smith D, et al. COVID19: Combining Antiviral and Anti-Inflammatory Treatments. Lancet Infect Dis (2020) 20(4):400-2. doi: 10.1016/S1473-3099(20)30132-8

101. Stebbing J, Sánchez Nievas G, Falcone M, Youhanna S, Richardson P, Ottaviani S, et al. JAK Inhibition Reduces SARS-CoV-2 Liver Infectivity and Modulates Inflammatory Responses to Reduce Morbidity and Mortality. Sci $A d v$ (2021) 7(1):eabe4724. doi: 10.1126/sciadv.abe4724

102. Hammarén HM, Virtanen AT, Raivola J, Silvennoinen O. The Regulation of JAKs in Cytokine Signaling and its Breakdown in Disease. Cytokine (2019) 118:48-63. doi: 10.1016/j.cyto.2018.03.041

103. Cantini F, Niccoli L, Nannini C, Matarrese D, Natale MED, Lotti P, et al. Beneficial Impact of Baricitinib in COVID-19 Moderate Pneumonia; Multicentre Study. J Infect (2020) 81(4):647-79. doi: 10.1016/j.jinf.2020.06.052

104. Bronte V, Ugel S, Tinazzi E, Vella A, De Sanctis F, Canè S, et al. Baricitinib Restrains the Immune Dysregulation in Patients With Severe COVID-19. J Clin Invest (2020) 130(12):6409-16. doi: 10.1172/JCI141772

105. Petrone L, Petruccioli E, Alonzi T, Vanini V, Cuzzi G, Najafi Fard S, et al. InVitro Evaluation of the Immunomodulatory Effects of Baricitinib: Implication for COVID-19 Therapy. J Infect (2021) 82(4):58-66. doi: 10.1016/j.jinf.2021.02.023

106. Vannucchi AM, Sordi B, Morettini A, Nozzoli C, Poggesi L, Pieralli F, et al. Compassionate Use of JAK1/2 Inhibitor Ruxolitinib for Severe COVID-19: A Prospective Observational Study. Leukemia (2021) 35(4):1121-33. doi: 10.1038/s41375-020-01018-y

107. Cao Y, Wei J, Zou L, Jiang T, Wang G, Chen L, et al. Ruxolitinib in Treatment of Severe Coronavirus Disease 2019 (COVID-19): A Multicenter, Single-Blind, Randomized Controlled Trial. J Allergy Clin Immunol (2020) 146(1):137-146.e3. doi: 10.1016/j.jaci.2020.05.019

108. Kalil AC, Patterson TF, Mehta AK, Tomashek KM, Wolfe CR, Ghazaryan V, et al. Baricitinib Plus Remdesivir for Hospitalized Adults With Covid-19. N Engl J Med (2021) 384(9):795-807. doi: 10.1056/NEJMoa2031994

109. Marconi VC, Ramanan AV, de Bono S, Kartman CE, Krishnan V, Liao R, et al. Efficacy and Safety of Baricitinib for the Treatment of Hospitalised Adults With COVID-19 (COV-BARRIER): A Randomised, Double-Blind, Parallel-Group, Placebo-Controlled Phase 3 Trial. Lancet Respir Med (2021) 9:1407-18. doi: 10.1016/S2213-2600(21)00331-3

110. Wu D, Yang XO. TH17 Responses in Cytokine Storm of COVID-19: An Emerging Target of JAK2 Inhibitor Fedratinib. J Microbiol Immunol Infect (2020) 53(3):368-70. doi: 10.1016/j.jmii.2020.03.005

111. Guimarães PO, Quirk D, Furtado RH, Maia LN, Saraiva JF, Antunes MO, et al. Tofacitinib in Patients Hospitalized With Covid-19 Pneumonia. N Engl J Med (2021) 385(5):406-15. doi: 10.1056/NEJMoa2101643

112. Cavalli G, De Luca G, Campochiaro C, Della-Torre E, Ripa M, Canetti D, et al. Interleukin-1 Blockade With High-Dose Anakinra in Patients With COVID-19, Acute Respiratory Distress Syndrome, and Hyperinflammation: A Retrospective Cohort Study. Lancet Rheumatol (2020) 2(6):e325-31. doi: 10.1016/S2665-9913(20)30127-2

113. Huet T, Beaussier H, Voisin O, Jouveshomme S, Dauriat G, Lazareth I, et al. Anakinra for Severe Forms of COVID-19: A Cohort Study. Lancet Rheumatol (2020) 2(7):e393-400. doi: 10.1016/S2665-9913(20)30164-8
114. Caricchio R, Abbate A, Gordeev I, Meng J, Hsue PY, Neogi T, et al. Effect of Canakinumab vs Placebo on Survival Without Invasive Mechanical Ventilation in Patients Hospitalized With Severe COVID-19: A Randomized Clinical Trial. JAMA (2021) 326(3):230-9. doi: 10.1001/ jama.2021.9508

115. Patel J, Beishuizen A, Ruiz XB, Boughanmi H, Cahn A, Criner GJ, et al. A Randomized Trial of Otilimab in Severe COVID-19 Pneumonia (OSCAR). medRxiv (2021) 04.14.21255475. doi: 10.1101/2021.04.14.21255475

116. Temesgen Z, Burger CD, Baker J, Polk C, Libertin CR, Kelley CF, et al. Lenzilumab in Hospitalised Patients With COVID-19 Pneumonia (LIVEAIR): A Phase 3, Randomised, Placebo-Controlled Trial. Lancet Respir Med (2021) 1:S2213-2600(21)00494-X. doi: 10.1016/S2213-2600(21)00494-X

117. Cremer PC, Abbate A, Hudock K, McWilliams C, Mehta J, Chang SY, et al. Mavrilimumab in Patients With Severe COVID-19 Pneumonia and Systemic Hyperinflammation (MASH-COVID): An Investigator Initiated, Multicentre, Double-Blind, Randomised, Placebo-Controlled Trial. Lancet Rheumatol (2021) 3(6):e410-8. doi: 10.1016/S2665-9913(21)00070-9

118. Rodríguez-Baño J, Pachón J, Carratalà J, Ryan P, Jarrín I, Yllescas M, et al. Treatment With Tocilizumab or Corticosteroids for COVID-19 Patients With Hyperinflammatory State: A Multicentre Cohort Study (SAM-COVID-19). Clin Microbiol Infect (2021) 27(2):244-52. doi: 10.1016/j.cmi.2020.08.010

119. Yuan M, Xu X, Xia D, Tao Z, Yin W, Tan W, et al. Effects of Corticosteroid Treatment for Non-Severe COVID-19 Pneumonia: A Propensity Score-Based Analysis. Shock (2020) 54(5):638-43. doi: 10.1097/SHK.0000000000001574

120. Campins L, Boixeda R, Perez-Cordon L, Aranega R, Lopera C, Force L. Early Tocilizumab Treatment Could Improve Survival Among COVID-19 Patients. Clin Exp Rheumatol (2020) 38(3):578.

121. Ramiro S, Mostard RLM, Magro-Checa C, van Dongen CMP, Dormans T, Buijs J, et al. Historically Controlled Comparison of Glucocorticoids With or Without Tocilizumab Versus Supportive Care Only in Patients With COVID19-Associated Cytokine Storm Syndrome: Results of the CHIC Study. Ann Rheum Dis (2020) 79(9):1143-51. doi: 10.1136/annrheumdis-2020-218479

122. Trouillet-Assant S, Viel S, Gaymard A, Pons S, Richard JC, Perret M, et al. Type I IFN Immunoprofiling in COVID-19 Patients. J Allergy Clin Immunol (2020) 146(1):206-8. doi: 10.1016/j.jaci.2020.04.029

123. Hung IF, Lung KC, Tso EY, Liu R, Chung TW, Chu MY, et al. Triple Combination of Interferon Beta-1b, Lopinavir-Ritonavir, and Ribavirin in the Treatment of Patients Admitted to Hospital With COVID-19: An OpenLabel, Randomised, Phase 2 Trial. Lancet (2020) 395(10238):1695-704. doi: 10.1016/S0140-6736(20)31042-4

124. Feld JJ, Kandel C, Biondi MJ, Kozak RA, Zahoor MA, Lemieux C, et al. Peginterferon Lambda for the Treatment of Outpatients With COVID-19: A Phase 2, Placebo-Controlled Randomised Trial. Lancet Respir Med (2021) 9 (5):498-510. doi: 10.1016/S2213-2600(20)30566-X

125. Dyavar SR, Singh R, Emani R, Pawar GP, Chaudhari VD, Podany AT, et al. Role of Toll-Like Receptor 7/8 Pathways in Regulation of Interferon Response and Inflammatory Mediators During SARS-CoV2 Infection and Potential Therapeutic Options. BioMed Pharmacother (2021) 141:111794. doi: 10.1016/j.biopha.2021.111794

126. Beigel JH, Tomashek KM, Dodd LE, Mehta AK, Zingman BS, Kalil AC, et al. Remdesivir for the Treatment of Covid-19 - Final Report. N Engl J Med (2020) 383(19):1813-26. doi: 10.1056/NEJMoa2007764

127. Chan N, Eikelboom J. Hypercoagulability and Thrombosis in COVID-19: A Modifiable Cause for Mortality? Eur Heart J (2021) 42(33):3143-5. doi: 10.1093/eurheartj/ehab417

128. Clausen TM, Sandoval DR, Spliid CB, Pihl J, Perrett HR, Painter CD, et al. SARS-CoV-2 Infection Depends on Cellular Heparan Sulfate and ACE2. Cell (2020) 183(4):1043-1057.e15. doi: 10.1016/j.cell.2020.09.033

129. Goletti D, Cantini F. Baricitinib Therapy in Covid-19 Pneumonia - An Unmet Need Fulfilled. N Engl J Med (2021) 384(9):867-9. doi: 10.1056/NEJMe2034982

Conflict of Interest: The authors declare that the research was conducted in the absence of any commercial or financial relationships that could be construed as a potential conflict of interest.

Publisher's Note: All claims expressed in this article are solely those of the authors and do not necessarily represent those of their affiliated organizations, or those of the publisher, the editors and the reviewers. Any product that may be evaluated in 
this article, or claim that may be made by its manufacturer, is not guaranteed or endorsed by the publisher.

Copyright $\odot 2022$ Zizzo, Tamburello, Castelnovo, Laria, Mumoli, Faggioli, Stefani and Mazzone. This is an open-access article distributed under the terms of the
Creative Commons Attribution License (CC BY). The use, distribution or reproduction in other forums is permitted, provided the original author(s) and the copyright owner(s) are credited and that the original publication in this journal is cited, in accordance with accepted academic practice. No use, distribution or reproduction is permitted which does not comply with these terms. 